\title{
Value Preserving Welfare Weights for Social Optimization Problems*
}

\author{
Alexis Anagnostopoulos, Eva Carceles-Poveda, Yair Tauman
}

September 5, 2016

\begin{abstract}
Social optimization problems maximize the weighted sum of individual utilities over feasible allocations that satisfy certain constraints. In this paper, we propose a method that determines endogenous individual weights for such problems. We first provide three axioms which uniquely determine, for any welfare function, the contribution of a bundle of goods to that welfare function. We then define weights to be value preserving (VP) if the contribution of an individual's initial endowments to the social welfare function is proportional to the contribution of the final consumption allocation to that individual's welfare function. That is, the selected weights set the ratio of the social value of one's endowment to the private value of one's final allocation equal across individuals. We show that VP weights coincide with Negishi weights and the corresponding VP allocations coincide with Walrasian allocations in Arrow-Debreu economies. In contrast to Negishi weights, VP weights can also be used in economies with distortionary taxes or incomplete markets where the welfare theorems do not hold. As an application, we show how VP weights can be computed numerically in the context of a standard optimal taxation problem. We also compare the optimal tax scheme under VP weights with the one under exogenously assumed equal weights. By muting the redistribution motive inherent in the equal weights assumption, VP weights can highlight the aspects of optimal tax schemes that derive from taxation motives other than redistribution. Finally, we show how to extend our general methodology for computing social welfare weights to other normative principles of justice.
\end{abstract}

Keywords: Social Welfare Function, Heterogeneous Households, Redistribution, Optimal Taxation

JEL Classification: D63, E62, H21

${ }^{*}$ We would like to thank Marina Azzimonti, Dirk Krueger, Fransesc Ortega, Victor Rios-Rull, Warren Sanderson and Matthew Weinzierl for useful comments and suggestions. 


\section{INTRODUCTION}

A typical social optimization problem maximizes the weighted sum of individual utilities over feasible allocations that satisfy certain constraints. Such problems are often used to understand the properties of constrained efficient allocations and to characterize optimal government policy. The purpose of this paper is to provide a method that can be used to determine endogenously the set of individual weights for any social optimization problem.

When the fundamental welfare theorems hold, Negishi (1960) provides a method which endogenously determines welfare weights by requiring that the resulting allocation coincides with the Walrasian allocation. Although the Negishi method was not developed with a view to achieving justice, some authors have argued that market allocations are indeed just. ${ }^{1}$ Mankiw's (2010) Just Deserts Theory is a recent example. An alternative view on achieving fair allocations calls for choosing equal weights (EW) for every agent, which is often justified on the grounds of justice behind the veil of ignorance. ${ }^{2}$ This alternative is commonly used in modern macroeconomics where the environments incorporate frictions such as distortionary taxation or incomplete markets and the Negishi approach is not available. In this paper, we suggest an alternative to the EW approach that is closer to the Just Deserts view of justice and that can be applied to environments where the fundamental welfare theorems do not hold. Specifically, the paper defines value preserving (VP) weights to be weights which ensure that the value of an individual's contribution to social welfare is in proportion to the value the individual receives in the final allocation of the social optimization problem.

We first characterize axiomatically a mechanism that allows us to compute the per unit contribution of a good to a welfare function. The mechanism we propose is uniquely characterized by a set of three axioms. Given a bundle of commodities, the first axiom (rescaling) requires that the per unit contribution of a commodity should be independent of the units of measurement. The second axiom (separability) asserts that if the welfare function can be decomposed into the sum of different welfare functions, each of which is generated by different and disjoint sets of commodities, then the per unit contribution of a commodity depends only on the corresponding welfare function. The third axiom (continuity) requires that the mechanism is continuous in a neighborhood of both the welfare function and the initial bundle. It is shown that the unique mechanism that satisfies these three axioms equates the per unit contribution of a commodity to the marginal welfare with respect to that commodity.

We use this mechanism in defining the contribution of a bundle of goods to a welfare function as the inner product of the per unit contributions and the bundle. In turn, this provides a way to measure, for any set of individual welfare weights, the contribution of an individual's initial endowment of goods to social welfare (social value) and the contribution of the individual's final allocation of goods to his individual welfare (individual value). The VP weights are the ones that equate the ratio of individual to social contributions across

\footnotetext{
${ }^{1}$ There is a wide range of views on what constitutes a fair approach. For earlier references see Rawls (1971), Noszick (1974) and Aumann (1975). Saez and Stantcheva (2014), Fleurbaey and Maniquet (2014) and Weinzierl (2015) provide authoritative reviews of both classic and recent contributions to that literature.

${ }^{2}$ The argument is based on Harsanyi's $(1953,1955)$ early contributions. However, Weinzierl (2014) has recently presented survey evidence indicating that only a minority of individuals consider the EW approach and its implications for taxation as fair.
} 
agents.

Our second contribution lies in the characterization of the VP weights and allocations. We show that VP weights coincide with Negishi weights, and the corresponding VP allocations coincide with Walrasian allocations, for economies in which the welfare theorems hold. Intuitively, the reason is that, in such environments, the gradient of the welfare function at the initial bundle is a competitive equilibrium price vector. Hence, the social value of an individual's endowments coincides with the market value of his initial endowments and, by the budget constraint, also equals the value of his final allocation. This result provides some support for Mankiw's argument regarding the justice of market allocations. Notice, however, that the result is only true in a frictionless environment and will not, in general, hold in the presence of frictions. Nevertheless, VP weights and allocations can still be computed for economies in which the equilibrium is not Pareto optimal, such as economies with incomplete markets or distortionary taxes, where the Negishi method is not applicable. In this sense, our approach can be seen as a natural extension of the Negishi method to these types of economies. A crucial property of the VP approach is that the allocations resulting from the VP weights are invariant under rescaling of the units of utilities. That is, a positive affine transformation of one (or more) individual utility does not change the set of VP allocations. This stands in sharp contrast with the EW approach which has the undesirable property that a rescaling of utilities changes the prescribed allocation.

The VP approach and the EW approach make implicit assumptions regarding the social preference for equality and redistribution that stand, in some sense, at opposite extremes. We illustrate this in the context of an application to optimal taxation. We first show that under VP weights a government that needs to raise revenue and can use non-distortionary taxes, raises revenues from each individual in proportion to the present (market) value of their initial endowments. This clarifies the sense in which the VP approach mutes the redistribution motive and is contrasted to the EW approach which, in that example, prescribes taxes that implement perfect equalization of wealth and consumption across individuals.

To understand better the properties of VP allocations in the presence of distortions we also provide a numerical example with distortionary taxation and incomplete markets. For this we use a stylized, two-period model where the government's tax instruments are limited to flat rate taxes on capital income and labor income. The example is constructed so that the labor tax is non-distortionary, whereas the capital tax distorts the saving decisions of individuals. This provides a useful complete markets benchmark since, in this case, labor income taxes would be the efficient means of raising all revenue in the absence of any equity motive. We show that, with initial wealth inequality, the social preference for redistribution can have significant effects on the optimal tax scheme. With equal weights, the optimal capital tax rate increases, and the labor tax rate decreases, as inequality increases. Even moderate levels of inequality can result in a tax rate that is higher on capital income than labor income purely for redistribution reasons and despite the distortions introduced. Interestingly, under VP weights capital taxes also increase with inequality. The reason is that if all revenues were financed through labor income taxes, it would imply (reverse) redistribution and violate the VP principle. However, capital tax rates rise more slowly with inequality 
and do not become higher than labor taxes even when the wealth-poor hold no capital at all. When we introduce uninsured idiosyncratic risk a la Aiyagari (1994), taxing labor income can have the benefit of reducing risk. This insurance motive for taxation calls for high labor taxes and low capital taxes and goes against the redistribution motive. We argue that the VP approach can serve as a useful way to disentangle the redistribution motive from the insurance motive. ${ }^{3}$

Our paper proposes two distinct, novel ideas: a method to measure value (contribution mechanism) and a normative principle (VP). We discuss alternatives to each of those ideas after the numerical example. Regarding normative principles, two commonly used principles are the equal sacrifice principle and the Rawlsian principle. We show how to formalize these two alternative principles and compute the corresponding social welfare weights using our contribution mechanism. Conversely, we also discuss how the VP principle can be formalized using alternative contribution mechanisms. In particular, we provide a family of contribution mechanisms that computes the weighted average marginal value of endowments and which includes our mechanism as a special case in which all the weight is concentrated at one point. We show that, if utilities are homogeneous, each one of those coincides with our contribution mechanism. We also discuss how to compute the contribution of individuals to welfare using the Shapley (1953) value.

Our paper is related to, and establishes a link between, several distinct strands of literature. It is motivated by the macroeconomic literature that uses social welfare functions in environments where the welfare theorems do not hold because of market incompleteness or tax distortions. In this literature, it is common practice to use the sum of individual utilities, with equal weights, as the social objective. ${ }^{4}$ Instead, we obtain endogenous welfare weights using the value preserving principle. ${ }^{5}$ We use a standard optimal taxation example to show how VP weights can be computed (in some cases analytically) and to illustrate that the choice of weights can be crucial for the conclusions offered in that literature.

In more recent contributions on optimal taxation, Heathcote, Storesletten and Violante (2014), Weinzierl (2014), Saez and Stantcheva (2015) and Fleurbaey and Maniquet (2014) have considered alternatives to the equal weights approach. ${ }^{6}$ Heathcote et al (2014) present an analytically tractable model with several frictions including incomplete markets, distortionary taxation and externalities. They use the Negishi weights from the corresponding first best economy to determine the optimal tax progressivity in their environment with

\footnotetext{
${ }^{3}$ Throughout the paper, we use the term redistribution to refer to ex-ante redistribution and distinguish this from insurance which can be thought of as redistribution after the resolution of uncertainty.

${ }^{4}$ Aiyagari (1995), Domeij and Heathcote (2004), Abraham and Carceles-Poveda (2010) and Anagnostopoulos et al (2012) use this social objective for taxation problems. Davila et al (2012) use it to compare the constrained efficient allocations to the competitive equilibrium allocations in an economy with incomplete markets.

${ }^{5}$ Endogenous and time varying Pareto weights are also computed in the literature on endogenous incomplete markets arising from the presence of limited commitment (see for example Kehoe and Perri (2002). In that literature, however, the set of initial Pareto weights are exogenously given and it is exactly those initial weights that our approach aims to determine endogenously.

${ }^{6}$ In an earlier contribution, Benabou (2002) uses a social welfare function that focuses on efficiency and abstracts from equity concerns to study taxation and education policy.
} 
frictions. In contrast, our VP weights depend on the nature of the friction because they take into account the fact that the value of an endowment can change depending on the friction. We view our method as an extension of Negishi to environments with frictions. Weinzierl (2014) presents survey evidence which provides support for the incorporation of the principle of equal sacrifice into the social objective. In an application, he uses a social welfare function which includes a utilitarian component but penalizes deviations from equal sacrifice and shows that such an objective can explain a number of features of US tax policy. In contrast we follow a normative approach and we consider a different principle. Crucially, because we use our contribution mechanism to measure value, our approach yields allocations that are invariant under non-uniform affine transformations of utilities. This stands in contrast to existing applications of the sacrifice principle where utility levels are used to measure value. These have the undesirable feature that allocations change when a constant is added to utilities or when utilities are multiplied by a positive scalar. We briefly discuss how our contribution mechanism can be used to avoid this issue even when the equal sacrifice principle is used.

Saez and Stantcheva (2015) propose a non welfarist approach to optimal tax theory that can accommodate different principles or redistribution preferences. In particular, the authors derive optimal taxes by applying marginal social welfare weights, that are inspired by different fairness principles, directly to earning levels. Instead, Fleurbaey and Maniquet (2014) show that one can incorporate different fairness principles into the standard social welfare framework to do optimal taxation by treating utilities as normative indices that embed these ethical principles. Our work is complementary to these two papers. We use a welfarist approach but obtain the implications for optimal taxation for a specific principle that takes a stand on social preferences about redistribution. As stated earlier, the VP principle we propose is closely related to Mankiw (2010), who argues that an individual's compensation should reflect his or her social contribution, but without providing an explicit formalization. Our paper can be viewed as a formalization of this idea.

The VP principle is also related to the literature on values of cooperative games, even though our approach is not game theoretical. Shapley (1969) determines endogenously individual weights to enable the interpersonal comparison of utilities in finite cooperative games with non-transferable utilities. For a pure exchange complete market economy, these weights equate for every trader his contribution to the welfare of the grand coalition (i.e. his Shapley (1953) value ) with the weighted utility he obtains from the optimal final allocation of the resources of the grand coalition. The resulting allocation is called a value allocation. Aumann (1975) uses a similar idea to determine individual weights endogenously for non-atomic pure exchange economies. ${ }^{7}$ He proves, for differentiable utilities, that the set of value allocations coincides with the set of Walrasian allocations, and hence the proposed weights coincide with the Negishi weights. This result only holds for non atomic pure exchange economies with complete markets and no production. Our approach is different. We measure the value of a trader via the contribution of his initial endowments to the total welfare without the use of

\footnotetext{
${ }^{7}$ In Aumann (1975), the value used is an axiomatic extension of the Shapley value for non-atomic games derived in Aumann and Shapley (1974)
} 
any game theoretic notion. The equivalence between Walrasian and VP allocations that we derive holds for any Arrow-Debreu complete market economy, with or without production, and with a finite number of agents or a continuum of agents with a finite number of types.

Finally, our axiomatic approach is related to the literature on cost allocation as in Mirman and Tauman (1982) and Samet and Tauman (1982). The latter is the one most closely related to our work. The authors characterize axiomatically the marginal cost pricing mechanism for continuously differentiable functions that contain no fixed costs component. Our axiomatization differs from theirs in several respects. To accommodate utility functions that are widely used in the macroeconomic literature, we allow for welfare functions that do not necessarily vanish at zero and we also allow for utilities that need not be continuously differentiable at zero. The most fundamental difference, however, is in the set of axioms. Their approach relies on the axiom of additivity which in our context would assert that the contribution of a bundle of goods to the sum of two welfare functions is the sum of the contribution of that bundle to each of them separately. This axiom can be justified as an accounting convention in the context of cost functions, but it is harder to justify in the context of welfare functions. We dispose of the additivity axiom and the consistency axiom and replace them by the more appealing separability axiom. ${ }^{8}$

The paper is organized as follows. Section 2 defines in general terms the social optimization problem we are interested in and Section 3 defines and discusses the VP principle. Section 4 presents the axiomatic approach to define the contribution of a bundle of goods to a welfare function and proves the main theorem. Section 5 provides a characterization of the value preserving weights for an Arrow-Debreu economy. Section 6 provides an application of the theory to an optimal taxation example. Section 7 discusses alternative principles and contribution mechanisms and Section 8 summarizes and concludes.

\section{The Economy}

Consider an economy with $L$ goods that are indexed by $l \in\{1, \ldots, L\}, I$ agents indexed by $i \in\{1,2, \ldots, I\}$ and $J$ firms that are indexed by $j \in\{1,2, \ldots, J\} .{ }^{9}$ Let $\mathbb{R}^{L}$ be the $L$ dimensional Euclidean space, let $\mathbb{R}_{+}^{L}$ be the nonnegative orthant of $\mathbb{R}^{L}$ and let $\mathbb{R}_{++}^{L}$ be the positive orthant of $\mathbb{R}^{L}$. We let $w^{i}=\left(w_{1}^{i}, \ldots w_{L}^{i}\right) \in \mathbb{R}_{+}^{L}$ be the vector of initial good allocations (or endowments) of agent $i \in I$ and $w=\left(w^{1}, \ldots, w^{I}\right) \in \mathbb{R}_{+}^{L I}$ be the vector of initial good allocations. Similarly, $x^{i}=\left(x_{1}^{i}, \ldots, x_{L}^{i}\right) \in \mathbb{R}_{+}^{L}$ represents the vector of final allocations of agent $i$ and $x=\left(x^{1}, \ldots, x^{I}\right) \in \mathbb{R}_{+}^{L I}$ is the vector of final allocations.

A firm is identified with a production plan $z^{j} \in \mathbb{R}^{L}$. Let $z=\left(z^{1}, \ldots, z^{J}\right) \in \mathbb{R}^{L J}$ and let $\theta_{j}^{i} \in \mathbb{R}_{+}$be the initial share of agent $i$ in firm $j$ so that $\sum_{i=1}^{I} \theta_{j}^{i}=1$ for all $j$. Finally, let $u_{i}\left(x^{i}\right)$ be the utility function of agent $i$. It is assumed that each $u_{i}$ is continuous on $\mathbb{R}_{+}^{L}$.

Definition 1. Social Optimization Problem. A social optimization problem (SOP) is one that maximizes the weighted sum of utilities under constraints, where the individual

\footnotetext{
${ }^{8}$ From a technical perspective, we also provide a much simpler proof that does not rely on the Riesz representation theorem.

${ }^{9}$ In order to reduce notation we also use $L$ to denote the set of goods $\{1, \ldots, L\}$. We follow a similar convention for $I$ and $J$ throughout the paper.
} 
weights are $\lambda=\left(\lambda_{i}\right)_{i \in I}, \lambda_{i} \geq 0$ for all $i$ and $\sum_{i=1}^{I} \lambda_{i}=1$. The resulting value function $F_{\lambda}(w): \mathbb{R}_{+}^{L I} \rightarrow \mathbb{R}$ is a social welfare function (SWF) provided that the maximum exists, which is the case if utilities are continuous and the constraints define a compact subset of $\mathbb{R}_{+}^{L I} \times \mathbb{R}_{+}^{L J}$ for every $w \in \mathbb{R}_{+}^{L I}$. Formally,

$$
\begin{aligned}
F_{\lambda}(w) & \equiv \max _{x, z} \sum_{i=1}^{I} \lambda_{i} u_{i}\left(x^{i}\right) \text { s.t. } \\
x^{i} & \in \mathbb{R}_{+}^{L}, i=1, \ldots, I \\
g_{s}(x, z, w) & \geq 0, s=1, \ldots, S
\end{aligned}
$$

where $g_{s}: \mathbb{R}_{+}^{L I} \times \mathbb{R}_{+}^{L J} \times \mathbb{R}_{+}^{L I} \rightarrow \mathbb{R}$. The inequalities $g_{s}(x, z, w) \geq 0$ are constraints on the economy and the sets $\left\{(x, z, w) \mid g_{s}(x, z, w) \geq 0\right.$ for all $\left.s\right\}$ are compact.

For a simple example consider a pure exchange economy with complete markets. The social welfare function in such a setting will be

$$
\begin{aligned}
F_{\lambda}(w) & =\max _{\left(x^{i}\right)_{i \in I}} \sum_{i=1}^{I} \lambda_{i} u_{i}\left(x^{i}\right) \\
x^{i} & \in \mathbb{R}_{+}^{L}, i=1, \ldots, I \\
\sum_{i=1}^{I} x^{i} & \leq \sum_{i=1}^{I} w^{i}
\end{aligned}
$$

In this case the social welfare function depends only on the aggregate endowments $W \equiv$ $\sum_{i=1}^{I} w^{i} \in \mathbb{R}_{+}^{L}$ so that one could write instead an alternative welfare function $G_{\lambda}(W)$ with $G_{\lambda}: \mathbb{R}_{+}^{L} \rightarrow \mathbb{R}$. In general, $F_{\lambda}$ depends on the distribution of endowments $w$ and not just on the aggregate endowment $W$, that is, goods will have to be treated as differentiated according to who holds them. This example is also particular in the sense that the constraints are linear whereas in other SOPs, the constraints might not be linear or even convex and the social welfare function may not be concave.

\section{Value Preserving (VP) Welfare Weights}

Our objective is to determine welfare weights $\lambda$ or, equivalently, to choose one out of the constrained Pareto optimal allocations. The approach we propose is inspired by Aumann (1975) and Shapley (1969) who select weights so that each individual's allocation is related to their contribution to society. It differs substantially from the aforementioned papers in terms of how this contribution is measured and in terms of how the weights are chosen.

We measure the social value or social contribution of agents through their initial endowments, i.e. through what they bring with them. Similarly, the value of a final allocation of an individual is the contribution of the allocated bundle to their individual welfare. The next section provides a mechanism that can be used to compute the contribution of a bundle of goods to a welfare function, regardless of whether it is an individual utility function or a social welfare function. In this section, we take such a mechanism as given and describe the value preserving principle.

Denote $\tilde{w}^{i}=\left(0, \ldots, 0, w^{i}, 0, . ., 0\right) \in \mathbb{R}_{+}^{L I}$ and notice that $\tilde{w}^{i} \leq w$ and $\sum_{i} \tilde{w}^{i}=w$. Let $\lambda=\left(\lambda_{i}\right)_{i \in I}$ and let $C\left(F_{\lambda}, \tilde{w}^{i}, w\right)$ be the contribution of the bundle $\tilde{w}^{i}$ to the social welfare 
function $F_{\lambda}$ generated by the weights $\lambda$ and the vector of initial bundles $w$. Similarly, the contribution of the bundle $x^{i}$ to individual $i$ 's welfare function $\lambda_{i} u_{i}$ generated by the bundle $x^{i}$ is $C\left(\lambda_{i} u_{i}, x^{i}, x^{i}\right)$. In this case, we can simplify notation and write $C\left(\lambda_{i} u_{i}, x^{i}\right) .{ }^{10}$ Given the private and social contributions, welfare is allocated so that the value of the final allocation to an individual is proportional to the value of the individual's initial endowment bundle to society.

Definition 2. Value Preserving (VP) Weights and Allocations. Consider a SOP with weights $\lambda=\left(\lambda_{i}\right)_{i \in I}$, let $x_{\lambda} \in \mathbb{R}_{+}^{L I}$ be a maximizer and $F_{\lambda}(w)$ be the corresponding maximized value. The weights $\lambda=\left(\lambda_{i}\right)_{i \in I}$ and the final bundle allocation $x_{\lambda}$ are value preserving iff

$$
\frac{C\left(\lambda_{i} u_{i}, x_{\lambda}^{i}\right)}{C\left(F_{\lambda}, \tilde{w}^{i}, w\right)}=\frac{C\left(\lambda_{h} u_{h}, x_{\lambda}^{h}\right)}{C\left(F_{\lambda}, \tilde{w}^{h}, w\right)} \text { for all } i, h \in I
$$

The contribution of the initial allocation $\tilde{w}^{i}$ of agent $i \in I$ to social welfare is $C\left(F_{\lambda}, \tilde{w}^{i}, w\right)$. Similarly, the contribution of the final bundle $x_{\lambda}^{i}$ to the private welfare function $\lambda_{i} u_{i}$ is $C\left(\lambda_{i} u_{i}, x_{\lambda}^{i}\right)$. Thus, under $\mathrm{VP}$ weights, the ratio of the social to the private contribution is equalized across all individuals. To put it differently, the private contribution of an individual is proportional to his social contribution, where the proportion is the same for all individuals.

In the following section, we characterize the contribution mechanism by a set of three axioms. Subsequently, we show that, for Arrow-Debreu complete market economies, VP weights coincide with the Negishi weights and VP allocations coincide with competitive equilibrium allocations. Thus, our principle can be thought of as weighing individuals according to what they could obtain through voluntary trade.

There is a range of views on whether such a principle can be thought of as just. ${ }^{11}$ Recently, Mankiw (2010) has argued, in the libertarian tradition, that this is indeed the case. Referring to a similar principle, Aumann (1975) suggests instead the term "reasonable compromise" as opposed to "equitable solution". A very different perspective underlies the commonly used approach of assigning equal weights to all individuals, which is often justified using an argument based on the original position 'behind the veil of ignorance'. ${ }^{12}$ Our paper does not attempt to offer new arguments in favor of libertarian principles. Heterogeneity in endowments and utilities is assumed exogenously and any concept of justice would necessarily have to address the causes of this initial heterogeneity. The VP principle could be deemed just if one believed that all of the initial heterogeneity is deserved. Equal weights would be easier to justify if all heterogeneity were due to luck. We view these two assumptions regarding initial heterogeneity as the two extremes of a spectrum. The equal weights approach has been extensively used in macroeconomics. VP weights formalize an alternative extreme.

Mechanically, condition (2) provides equations that can be used to endogenously solve for the weights $\lambda$. Alternative principles, such as the equal sacrifice principle or the Rawlsian

\footnotetext{
${ }^{10}$ We have assumed that the individual welfare function depends only on $x^{i}$ and not any $x^{h}, h \neq i$. It would be straightforward to define $u_{i}$ on $\mathbb{R}_{+}^{L I}$, i.e. to allow for individual utilities that depend on the whole distribution $x$. In that case, the contribution would be denoted $C\left(\lambda_{i} u_{i}, \tilde{x}^{i}, x\right)$.

${ }^{11}$ See the introduction and, specifically, footnote 1.

${ }^{12}$ See Harsanyi $(1953,1955)$.
} 
principle, which could be used instead of the VP principle to determine weights are discussed in Section 7. We note here that the VP principle differs from the principle of equal sacrifice used by Weinzierl (2014) and, earlier by Young $(1988,1990)$ in a fundamental way: private and social values are defined for the same economy. In contrast, the equal sacrifice principle defines sacrifice by comparing allocations to a benchmark economy that is different than the economy in question. This introduces a certain level of arbitrariness in the choice of how to define the benchmark economy. For example, in tax applications this could be the economy with frictions but without a government or it could be the frictionless economy. Our approach avoids this difficult choice because it does not rely on such a benchmark economy.

\section{Axiomatic Approach}

In this section, we introduce an axiomatic approach to characterize the per unit contribution of a good to a welfare function. Let $m$ be the number of goods and let $w=\left(w_{1}, \ldots, w_{m}\right) \in \mathbb{R}_{+}^{m}$ denote a bundle of these goods. ${ }^{13}$ Let $(x, y) \in \mathbb{R}^{m} \times \mathbb{R}^{m}$. We write $x>>y$ iff $x_{l}>y_{l}$ for all $1 \leq l \leq m$. We write $x>y$ iff $x_{l} \geq y_{l}$ for all $1 \leq l \leq m$ and $x_{l}>y_{l}$ for at least one $l$. We write $x \geq y$ iff $x_{l} \geq y_{l}$ for all $1 \leq l \leq m$. Let $\mathcal{F}^{m}$ be the set of all real valued functions $F$ which are continuously differentiable $(c d)$ on $\mathbb{R}_{+}^{m} \backslash\{0\}$.

Definition 3. Per unit Contribution Mechanism. A per unit contribution mechanism for commodity $j, 1 \leq j \leq m$, is a function $\widehat{C}_{j}(\cdot, \cdot)$ which associates with every integer $m \geq 1$, and every $(F, w), F \in \mathcal{F}^{m}, w \in \mathbb{R}_{+}^{m} \backslash\{0\}$ an element $\widehat{C}_{j}(F, w) \in \mathbb{R}$.

The function $\widehat{C}_{j}(F, w)$ measures the per unit contribution of the $j^{\text {th }}$ commodity to the welfare function $F$ when the overall bundle of goods is $w$. Next, we present three axioms that uniquely determine the per unit contribution of a good.

Axiom 1: Rescaling. Let $F \in \mathcal{F}^{m}$ and $G \in \mathcal{F}^{m}$. Suppose that

$$
F(x)=q G\left(r_{1} x_{1}, . ., r_{m} x_{m}\right)+c
$$

where $q \neq 0$ and $c$ are real numbers and $r \in \mathbb{R}_{++}^{m}$. Then for every $j, 1 \leq j \leq m$

$$
\widehat{C}_{j}(F, w)=q r_{j} \widehat{C}_{j}\left(G, r_{1} w_{1}, . ., r_{m} w_{m}\right)
$$

for all $w \in \mathbb{R}_{+}^{m} \backslash\{0\}$.

This axiom requires that the per unit contribution is independent of the units of measurement of the goods. Consider an economy where the only good is apples $(m=1)$, let $x$ denote apple allocations in kilograms and let $F(x)$ be the welfare function. Let $G(x)$ represent the same welfare as $F$ but with the argument $x$ measured in grams. In this example $r=1000$, $q=1, c=0$ and $F(x)=G(1000 x)$. Then, $\widehat{C}_{j}(F, w)$ is the per kilogram contribution of $w$ kilograms of apples to the welfare $F$ and $\widehat{C}_{j}(G, 1000 w)$ is the per gram contribution of $1000 w$ grams ( $=w$ kilograms) of apples to the welfare $G$. The axiom requires that the per

\footnotetext{
${ }^{13}$ This means the welfare function has $m$ arguments. To relate to the notation of the previous section, in the case of an individual welfare function, or in the case where the social welfare function depends only on aggregate endowments, $m=L$. When the social welfare function depends on the whole distribution of endowments, then $m=L I$.
} 
kilogram contribution of $w$ kilograms is the same as 1000 times the per gram contribution of $1000 w$ grams of apples

$$
\widehat{C}_{j}(F, w)=1000 \widehat{C}_{j}(G, 1000 w)
$$

We also allow for the rescaling of the units of utils (which measure the level of welfare). If we change every original util into $q$ new utils, the contribution in terms of the new utils should be $q$ times that of the original utils. For example, consider an economy in which the value of a welfare function $G$ is measured in dollars. Let $F$ represent the same welfare as $G$ except that $F$ is measured in cents, namely $F(x)=100 G(x)$. In this case, the axiom requires that $\widehat{C}_{j}(F, w)=100 \widehat{C}_{j}(G, w)$ for $1 \leq j \leq m$. Finally, since $F$ and $F+c$ represent the same welfare function, it is also required that the per unit contribution mechanism is invariant under a scalar shift of welfare functions.

Axiom 2 (Separability). Let $w \in \mathbb{R}_{+}^{m} \backslash\{0\}$ and let $\left(A_{r}\right)_{r=1}^{k}$ be a partition of $\{1, \ldots, m\}$ with cardinality $\left|A_{r}\right|=m_{r}$ and $\sum_{r=1}^{k} m_{r}=m$. Let $F \in \mathcal{F}^{m}$ and $F^{r} \in \mathcal{F}^{m_{r}}, 1 \leq r \leq k$. Suppose that for all $x \in \mathbb{R}_{+}^{m}$

$$
F(x)=F^{1}\left(x^{1}\right)+\ldots+F^{k}\left(x^{k}\right)
$$

where $x^{r}$ denotes the projection of $x$ on the coordinates of $A^{r}$. Then for every $1 \leq r \leq k$ and $j \in A^{r}$.

$$
\widehat{C}_{j}(F, w)=\widehat{C}_{j}\left(F^{r}, w^{r}\right)
$$

This axiom refers to the special case where the set of goods can be separated into groups that are independent from each other, in the sense that the effect of one group on the welfare function is independent from the effect of other groups. In a typical economic optimization problem, this happens when all utilities and constraints are separable with respect to different groups of goods. For example, consider the individual welfare function in a multi-period endowment economy with perishable goods, no trade and utility that is additively separable across time. Then each period's bundle of endowments adds to individual welfare (the sum over time of period utilities) only through that period's utility function. The axiom requires that, in this case, the per unit contribution of a good in a given period to the individual's welfare equals the per unit contribution of that good to that period's utility.

Let $X \subseteq R^{m}$ be a compact set and let $\|F\|_{X}^{1}$ represent the $C^{1}(X)$-norm of $F$, defined by

$$
\|F\|_{X}^{1}=\max _{x \in X}\left[|F(x)|+\sum_{j=1}^{m}\left|\frac{\partial F}{\partial x_{j}}(x)\right|\right]
$$

Definition 4. Let $F \in \mathcal{F}^{m}$ and $w \in \mathbb{R}_{+}^{m} \backslash\{0\}$. We say that $\widehat{C}_{j}(F, w)$ is continuous at $(F, w)$ if for every $\epsilon>0$ there exists $\delta>0$ and $\eta>0$ such that for all $\widetilde{w}, w_{j} \leq \widetilde{w}_{j} \leq w_{j}+\eta$, $1 \leq j \leq m$, and for all $G \in \mathcal{F}^{m}$

$$
\|F-G\|_{X(w, \eta)}^{1}<\delta \rightarrow\left|\widehat{C}_{j}(F, w)-\widehat{C}_{j}(G, \widetilde{w})\right|<\epsilon
$$


where $X(w, \eta)$ is the box defined by ${ }^{14}$

$$
X(w, \eta)=\Pi_{j=1}^{m}\left[w_{j}, w_{j}+\eta\right]
$$

Axiom 3: (Continuity). $\widehat{C}_{j}(\cdot, \cdot)$ is continuous at $(F, w)$ for all $F \in \mathcal{F}^{m}$ and $w \in$ $\mathbb{R}_{+}^{m} \backslash\{0\}$.

Theorem 1 asserts that there is a unique per unit contribution mechanism that satisfies the above three axioms up to a scalar multiplication.

Theorem 1. A per unit contribution mechanism $\widehat{C}_{j}(\cdot, \cdot)$ satisfies Axioms $1-3$ for $1 \leq j \leq$ $m$ iff there exists $\alpha \in \mathbb{R}_{++}$such that for all $m \geq 1,1 \leq j \leq m, F \in \mathcal{F}^{m}$ and $w \in \mathbb{R}_{+}^{m} \backslash\{0\}$

$$
\widehat{C}_{j}(F, w)=\alpha \frac{\partial F}{\partial x_{j}}(w)
$$

The (positive) constant $\alpha$ is universal, namely, it is the same constant for all pairs $(F, w)$. If $I: \mathbb{R} \rightarrow \mathbb{R}$ is the identity function $(I(x) \equiv x)$ then $\alpha=\widehat{C}(I, 1)$. By Theorem $1, \widehat{C}(F, w)=$ $\alpha \nabla F(w)$, where

$$
\widehat{C}(F, w)=\left(\widehat{C}_{1}(F, w), \ldots, \widehat{C}_{m}(F, w)\right)
$$

The theorem asserts that the per contribution of a good defined by (3) satisfies the three axioms and vice versa, any contribution mechanism that satisfies the three axioms must be given by (3). The proof of Theorem 1 is provided in Appendix A.

Even though the setup of the theorem makes no reference to markets, an interpretation that we find useful in what follows is to think of the gradient $\nabla F(w)$ of the welfare function as a vector of (shadow) prices.

Finally, we define the overall contribution of a bundle of goods as follows.

Definition 5. Contribution Mechanism. Define the set $D$ as follows:

$$
D=\left\{\left(F, w^{\prime}, w\right) \mid F \in \mathcal{F}^{m} \text { for some } m, w \in \mathbb{R}_{+}^{m} \backslash\{0\}, 0 \leq w^{\prime} \leq w\right\}
$$

A contribution mechanism is a function $C(\cdot, \cdot, \cdot)$ which associates with every $\left(F, w^{\prime}, w\right) \in$ $D$ an element $C\left(F, w^{\prime}, w\right) \in \mathbb{R}$, where

$$
C\left(F, w^{\prime}, w\right)=\sum_{j=1}^{m} w_{j}^{\prime} \widehat{C}_{j}(F, w)
$$

Note that the contribution mechanism $C\left(F, w^{\prime}, w\right)$ represents the overall contribution of the bundle $w^{\prime} \leq w$ to any welfare function $F \in \mathcal{F}^{m}$ when the initial bundle is $w \in \mathbb{R}_{+}^{m} \backslash\{0\}$.

\footnotetext{
${ }^{14}$ Since some of the coordinates of $w$ may be zero, we only consider the right neighbourhood of $w$.
} 


\section{Value Preserving Weights for Arrow-Debreu Economies}

When the fundamental welfare theorems hold, the Negishi approach yields welfare weights such that a Pareto optimal allocation chosen by maximizing a weighted sum of individual welfare functions (utilities) coincides with the Walrasian allocation. We show in this section that, in such environments, our approach also yields the Walrasian allocation. Thus, VP weights coincide with Negishi weights and VP allocations coincide with Walrasian allocations in Arrow-Debreu complete market economies. We prove this result for economies with homothetic technologies. The result can be extended to the general case of non-homothetic technologies with a straightforward modification of the VP concept which we discuss at the end of the section.

5.1. Competitive Equilibrium. Each firm $j$ has a convex production set $Z_{j} \subseteq \mathbb{R}^{L}$. A production plan $z^{j}$ belongs to the production set $Z_{j}$ iff $f_{j}\left(z^{j}\right) \leq 0$, where $f_{j}\left(z^{j}\right)$ summarizes the technological constraints. We assume that $f_{j}: \mathbb{R}^{L} \rightarrow \mathbb{R}$ is twice continuously differentiable, $f_{j}(0) \leq 0$ and $\nabla f_{j}\left(z^{j}\right)>>0$ for all $j \in J$. Each firm $j$ maximizes profits and solves:

$$
\max _{z^{j}} p z^{j} \text { s.t. } f_{j}\left(z^{j}\right) \leq 0
$$

where $p \in \mathbb{R}_{+}^{L}$ is the vector of commodity prices. Since $f_{j}$ is continuous, $f_{j}\left(z^{j}\right)=0$ for any maximizer $z^{j}$.

Each household is initially endowed with $w^{i} \in \mathbb{R}_{+}^{L}$ units of the goods and $\theta_{j}^{i} \geq 0$ shares of each firm $j$, with $\sum_{i=1}^{I} \theta_{j}^{i}=1$ for all $j \in J$. Each household's utility is represented by a twice continuously differentiable, strictly increasing and concave function $u_{i}: \mathbb{R}_{+}^{L} \rightarrow \mathbb{R}$. Household $i$ takes $p, w^{i}$ as well as $\theta_{j}^{i}, z^{j}$ for all $j \in J$ as given and solves:

$$
\begin{array}{r}
\max _{x^{i}} u_{i}\left(x^{i}\right) \text { s.t. } \\
p x^{i}=p w^{i}+\sum_{j=1}^{J} \theta_{j}^{i} p z^{j}
\end{array}
$$

The Lagrangian for this problem is:

$$
\mathcal{L}_{i}=u_{i}\left(x^{i}\right)+\mu_{i}\left(p w^{i}+\sum_{j} \theta_{j}^{i} p z^{j}-p x^{i}\right)
$$

Assuming an interior solution, the first order conditions for $i=1, \ldots, I$ with respect to $x_{l}^{i}$, $l=1, \ldots L$ imply:

$$
\frac{\partial u_{i}\left(x^{i}\right)}{\partial x_{l}^{i}}=p_{l} \mu_{i}
$$

Let $E$ be the set of all economies described above. For each $e \in E$, we describe next the family of corresponding social optimization problems. 
5.2. Social Optimization Problem. The corresponding social optimization problem with welfare weights $\lambda=\left(\lambda_{i}\right)_{i=1}^{I}$ is given by:

$$
\begin{aligned}
F_{\lambda}(w) & =\max _{x, z} \sum_{i=1}^{I} \lambda_{i} u_{i}\left(x^{i}\right) \text { s.t. } \\
\sum_{i=1}^{I} x^{i} & =\sum_{i=1}^{I} w^{i}+\sum_{j=1}^{J} z^{j} \\
f_{j}\left(z^{j}\right) & \leq 0 \text { for } j=1, \ldots, J
\end{aligned}
$$

The Lagrangian is:

$$
\mathcal{L}=\sum_{i=1}^{I} \lambda_{i} u_{i}\left(x^{i}\right)+\sum_{l=1}^{L} \eta_{l}\left(\sum_{i=1}^{I} w^{i}+\sum_{j=1}^{J} z_{l}^{j}-\sum_{i=1}^{I} x_{l}^{i}\right)+\sum_{j=1}^{J} \nu_{j} f\left(z^{j}\right)
$$

Denote the maximizing production plan by $z_{\lambda}(w)=\left(z_{\lambda}^{1}(w), \ldots, z_{\lambda}^{J}(w)\right)$ and the maximizing consumption allocation by $x_{\lambda}(w)=\left(x_{\lambda}^{1}(w), \ldots, x_{\lambda}^{I}(w)\right) .{ }^{15}$ Assuming again an interior solution, it must be that

$$
\frac{\partial u_{i}\left(x_{\lambda}^{i}(w)\right)}{\partial x_{l}^{i}}=\frac{\eta_{l}}{\lambda_{i}}
$$

5.3. Equivalence of VP and Negishi weights. Consider first constant returns to scale technologies so that firms make zero profits. That is, assume that $z \in Z_{j}$ implies $q z \in Z_{j}$ for all $q \in \mathbb{R}_{+}$. Proposition 1 states the result for such homothetic technologies.

Proposition 1. Consider an economy $e \in E$ and suppose that technologies are homothetic. Let $\Delta^{I}=\left\{\lambda \in \mathbb{R}_{+}^{I} \mid \sum_{i=1}^{I} \lambda_{i}=1\right\}$. Then, $\lambda \in \Delta^{I}$ is a value preserving weight iff it is a Negishi weight for the initial endowments $w=\left(w^{1}, \ldots, w^{I}\right) \in \mathbb{R}_{+}^{L I} \backslash\{0\}$ of the economy.

The proof is provided in Appendix A.

It is straightforward to extend this result to general technologies by extending the definition of the initial endowments. To ensure the equivalence of VP and Negishi weights even for environments where firms can have positive profits, one needs to include in the contribution of an individual not only their initial bundle of commodities $w^{i}$ but also their ownership of firms. The way to do this is to consider the equilibrium production plans of the firms $\left(z^{j}(w)\right)_{j=1}^{J}$, multiplied by the shares of individual $i$ in each firm $\left(\theta_{j}^{i}\right)_{j \in J}$ as part of that individual's initial endowments. To be precise, define the modified initial endowment of individual $i$ as

$$
y^{i} \equiv w^{i}+\sum_{j=1}^{J} \theta_{j}^{i} z^{j}(w), i \in I
$$

and let $\tilde{y}^{i}=\left(0, \ldots, 0, y^{i}, 0, . ., 0\right) \in \mathbb{R}_{+}^{L I}$. Then measure the contribution of $i$ to social welfare $F_{\lambda}(w)$ through these modified initial endowments $\tilde{y}^{i}$ so that this contribution is now

\footnotetext{
${ }^{15}$ Note that both the value $F_{\lambda}$ and the allocations $x_{\lambda}, z_{\lambda}$ are written as functions of the whole distribution of endowments $w$ in order to conform to the notation used in previous sections. Clearly, in this case, these can be written as functions of the aggregate endowment only.
} 
$C\left(F_{\lambda}, \tilde{y}^{i}, w\right)$. The value preserving weights $\lambda=\left(\lambda_{1}, \ldots, \lambda_{I}\right)$ are now the solution to:

$$
\frac{C\left(\lambda_{i} u_{i}, x_{\lambda}^{i}(w)\right)}{C\left(\lambda_{h} u_{h}, x_{\lambda}^{h}(w)\right)}=\frac{C\left(F_{\lambda}, \tilde{y}^{i}, w\right)}{C\left(F_{\lambda}, \tilde{y}^{h}, w\right)} \text { for all } i, h \in I
$$

With this modification, replacing $\tilde{w}^{i}=\left(0, \ldots, 0, w^{i}, 0, . ., 0\right)$ by $\tilde{y}^{i}$ it is easy to verify that Proposition 1 holds true and the VP weights coincide with the Negishi weights for production technologies that are convex, even if the technologies are not homothetic.

Proposition 1 implies that, in a first best environment a planner allocating goods to individuals by maximizing a weighted sum of utilities with VP (or Negishi) weights chooses a Walrasian allocation, i.e. individuals obtain an allocation that they can achieve through voluntary trade. One can compare such an allocation to another Pareto optimal allocation that arises from equal weights (EW) and can be justified by appealing to a "behind the veil of ignorance" argument. In the latter, the planner would equalize marginal utility of wealth across consumers under standard assumptions (see Mas-Colell et al (1995)). Clearly, the two methods are very different with regard to the desirability of redistribution and can lead to very different allocations.

Moving away from first best environments, it is standard practice amongst macroeconomists to take the equal weights approach, partly because the Negishi approach is not applicable. An important exception to this is Heathcote, Storesletten and Violante (2014) who compute the Negishi weights for a first-best version of their model and apply the same weights to their model which incorporates externalities, incomplete markets and distortionary taxes. ${ }^{16}$ This would be equivalent to the VP approach if the value of a good in the economy with frictions were the same as in the corresponding first best economy, i.e. equal to the competitive price in the frictionless economy. The VP approach differs because it incorporates the value of each good in the actual (distorted) economy, where the value is measured using the marginal increase in social welfare arising from an increase in the available good in question.

\section{An Application to Optimal Taxation}

This section uses variations of an optimal taxation problem to illustrate how our method can be applied in practice and to hint on the potential importance of the choice of weights for such problems by comparing the VP allocations to the case of equal weights. The structure of the model is standard in the optimal (Ramsey) taxation literature. First, the government announces and commits to a tax policy. Subsequently, and given the tax policy, households and firms make decisions in a standard competitive equilibrium model. We provide first a general result which characterizes optimal policy in a benchmark scenario where the government uses lump sum (non-distortionary) taxes. Subsequently, we consider a two period version of a standard growth model with heterogeneous agents. From an applied perspective, we are mainly interested in environments with distortionary capital and labor taxes and incomplete markets. For these we do not have analytical results so we resort to numerical computations Although the model is stylized, it is rich enough to allow the analysis of op-

\footnotetext{
${ }^{16}$ Other exceptions include Benabou (2002), Weinzierl (2014) and Saez and Stantcheva (2015).
} 
timal tax policy as an instrument for revenue-raising, insurance provision and redistribution while under the restrictions imposed by the loss of efficiency from distortionary taxation.

6.1. Lump Sum Taxation. Let $G \in \mathbb{R}_{+}^{L}$ be the total lump sum revenue needed to be collected by the government. Let $\widehat{\tau} \in \mathbb{R}^{L I}$ denote a tax policy, where $\widehat{\tau}_{l}^{i}$ is the lump sum tax (or transfer) of good $l$ for agent $i$. We first define the competitive equilibrium given a feasible tax policy $\widehat{\tau} \in \mathbb{R}^{L I}$ such that $\sum_{i \in I} \widehat{\tau}^{i}=G$.

Definition 3. Given $G$, a competitive equilibrium with respect to a lump sum tax policy $\widehat{\tau}$ is a tuple $(\widehat{x}, \widehat{z}, \widehat{\tau}, \widehat{p})$, where $\widehat{x}^{i} \in \mathbb{R}_{+}^{L}, \widehat{z}^{j} \in \mathbb{R}^{L}, \widehat{\tau}^{i} \in \mathbb{R}^{L}, \widehat{p} \in \mathbb{R}_{+}^{L}, i \in I, j \in J$ such that:

$$
\begin{aligned}
& \sum_{i \in I} \widehat{x}^{i}=\sum_{i \in I} w^{i}-G+\sum_{j=1}^{J} \widehat{z}^{j} \\
& f_{j}\left(\widehat{z}^{j}\right) \leq 0, \text { for all } j \in J \leq \\
& \sum_{i \in I} \widehat{\tau}^{i}=G \\
& \widehat{z}^{j} \text { maximizes } \widehat{p} z^{j} \text { over }\left\{z^{j} \mid f_{j}\left(z^{j}\right) \leq 0\right\}, j \in J \\
& \widehat{x}^{i} \text { maximizes } u_{i}\left(x^{i}\right) \text { over }\left\{x^{i} \in \mathbb{R}_{+}^{L} \mid \widehat{p} x^{i} \leq \widehat{p}\left(w^{i}-\widehat{\tau}^{i}+\sum_{j \in J} \theta_{i}^{j} \widehat{z}^{j}\right)\right\}, i \in I
\end{aligned}
$$

We refer to $\widehat{p}$ as a competitive price with respect to $\widehat{\tau}$.

For the study of the optimal taxation problem, we consider all feasible lump sum tax policies and their corresponding competitive equilibria. Namely, the planner faces the following set of constraints $\widehat{L}(G)$ on allocations:

$$
\widehat{L}(G)=\left\{(\widehat{x}, \widehat{z}) \mid \text { there exist } \widehat{\tau} \in \mathbb{R}^{I L} \text { and } \widehat{p} \in \mathbb{R}_{+}^{L} \text { s.t. }(\widehat{x}, \widehat{z}, \widehat{\tau}, \widehat{p}) \text { satisfies (6)-(10) }\right\}
$$

The social welfare function of the Ramsey taxation problem can then be defined as ${ }^{17}$

$$
\widehat{F}_{\lambda}(w)=\max _{x, z} \sum_{i \in I} \lambda_{i} u_{i}\left(x^{i}\right) \text { s.t. }(x, z) \in \widehat{L}(G)
$$

A weight $\lambda$ is a VP weight with respect to $\widehat{F}_{\lambda}(w)$ iff

$$
\frac{\nabla \widehat{F}_{\lambda}(w) \tilde{w}^{i}}{\nabla \widehat{F}_{\lambda}(w) \tilde{w}^{h}}=\frac{\lambda_{i} \nabla u_{i}\left(\widehat{x}_{\lambda}^{i}\right) \widehat{x}_{\lambda}^{i}}{\lambda_{h} \nabla u_{h}\left(\widehat{x}_{\lambda}^{h}\right) \widehat{x}_{\lambda}^{h}}, i, h \in I
$$

where $\widehat{x}_{\lambda}$ is a maximizer of (11).

As in Proposition 1, we maintain the assumptions of smooth and concave utilities, smooth and convex technologies as well as technologies that are homothetic. ${ }^{18}$

Proposition 2. Let $W-G>>0$ where $W=\sum_{i \in I} w^{i}$. A lump sum tax policy $\widehat{\tau}$ is a VP tax and $\lambda \in \Delta^{I}$ is the corresponding VP weight iff $\widehat{p} \widehat{\tau}^{i}=\frac{\widehat{p} w^{i}}{\widehat{p} W} \widehat{p} G$, where $\widehat{p}$ is the competitive price with respect to $\widehat{\tau}$.

\footnotetext{
${ }^{17}$ This formulation is known as the primal approach to the Ramsey taxation problem, where the government chooses allocations subject to implementability constraints.

${ }^{18}$ We can dispense with homotheticity by modifying the initial bundle as is done right after Proposition 1.
} 
The proof of Proposition 2 appears in the appendix. The proposition shows that VP taxes require each agent's overall tax liability to be proportional to that agent's share of initial wealth $\widehat{p} w^{i}$. It is easy to verify that

$$
\frac{\hat{p} w^{i}}{\hat{p} w^{h}}=\frac{\hat{p}\left(w^{i}-\hat{\tau}^{i}\right)}{\hat{p}\left(w^{h}-\hat{\tau}^{h}\right)}=\frac{\hat{p} x^{i}}{\hat{p} x^{h}}, i, h \in I
$$

which means that the before-tax and after-tax distributions of wealth (and, hence, expenditure) are the same. In the special case where the government does not need to raise revenue $(G=0), \widehat{\tau}^{i}=0$ for all $i$ is a VP tax. ${ }^{19}$

An Example with closed form solution. Consider an economy with two periods $t=1,2$, a continuum of identical firms and three traded goods in each period: a final (consumption) good, capital services and labor. In each period $t$, a representative firm rents capital $K_{t-1}$ and labor $N_{t}$ at competitive prices $R_{t}$ and $\omega_{t}$ and uses them as inputs to produce a consumption good $Y_{t}$ using a constant returns to scale technology

$$
Y_{t}=K_{t-1}^{\theta} N_{t}^{1-\theta}, t=1,2
$$

where $0<\theta<1$.

The economy is populated by a continuum of households represented by the interval $[0,1]$. Households are of two types which are indexed by $i=1,2$. A proportion $\pi_{i}$ of households is initially endowed with $k_{i 0}$ units of capital, with $\pi_{1}+\pi_{2}=1$. This is the only source of heterogeneity across the two types of households and the initial distribution of capital $\left(k_{10}, k_{20}\right)$ is given exogenously. ${ }^{20}$ Households are also endowed with $X_{i t}=X$ units of time in each period which can be allocated to labor or leisure. In each period, households decide on the supply of labor $n_{i t}$ and capital services $k_{i t}$ to that period's firm and on their demand for final goods. The final goods in period 1 can be consumed $c_{i 1}$ or saved $k_{i 1}$ (i.e. transformed one-to-one to capital services for period 2 production). In period 2 , the final goods bought are consumed $c_{i 2}$. Finally, the government needs to raise a revenue of $G_{1}$ units of the final good in period one and can use type-dependent lump sum taxes/transfers $\tau_{i}$. The period-by-period budget constraints of a household of type $i$ can thus be written as

$$
\begin{aligned}
c_{i 1}+k_{i 1} & =R_{1} k_{i 0}+\omega_{1} n_{i 1}-\tau_{i} \\
c_{i 2} & =R_{2} k_{i 1}+\omega_{2} n_{i 2}
\end{aligned}
$$

and the government budget is

$$
G_{1}=\pi_{1} \tau_{1}+\left(1-\pi_{1}\right) \tau_{2}
$$

Although we have specified the sequential trade version of the household budgets, complete markets ensure the date- 0 trade version is equivalent. As a result, this model can

\footnotetext{
${ }^{19}$ Note that the VP tax $\widehat{\tau}$ is not uniquely determined in this case unless the tax is only on one good. Every $\tau$ such that $\widehat{p} \widehat{\tau}^{i}=\widehat{p} \tau^{i}$ for all $i \in I$ is a VP tax as well.

${ }^{20}$ There are clearly many dimensions of household heterogeneity one could consider such as wealth, ability or preferences. Given the illustrative purpose of this application, we choose to focus on initial wealth heterogeneity only. We view this as a natural starting point given the abundance of existing work on taxation that focuses on this type of heterogeneity.
} 
be cast in the general Arrow-Debreu notation of the previous section. This is achieved by defining, for the representative of type $i$, endowments $w^{i}=\left(0,0, \pi_{i} k_{i 0}, 0, \pi_{i} X_{i 1}, \pi_{i} X_{i 2}\right)$, production plans $z^{1}=\left(Y_{1}, 0,-K_{0}, 0,-N_{1}, 0\right), z^{2}=\left(0, Y_{2}, 0,-K_{1}, 0,-N_{2}\right)$, allocations $x^{i}=$ $\left(\pi_{i}\left(c_{i 1}+k_{i 1}\right), \pi_{i} c_{i 2}, 0,-\pi_{i} k_{i 1}, \pi_{i}\left(X_{i 1}-n_{i 1}\right), \pi_{i}\left(X_{i 2}-n_{i 2}\right)\right)$ and policy $G=\left(G_{1}, 0,0,0,0,0\right)$, $\tau^{i}=\left(\pi_{i} \tau_{i}, 0,0,0,0,0\right)$. Using the fact that the final good at $t=1$ and the capital service at $t=2$ are the same good and normalizing their common price to one, the price vector can be written as $p=\left(1, \frac{1}{R_{2}}, R_{1}, 1, \omega_{1}, \frac{\omega_{2}}{R_{2}}\right)$. By Proposition 2 , it follows that the VP tax satisfies

$$
\tau_{i}^{V P}=\frac{R_{1} k_{i 0}+\omega_{1} X_{i 1}+\frac{\omega_{2}}{R_{2}} X_{i 2}}{R_{1} K_{0}+\omega_{1} X+\frac{\omega_{2}}{R_{2}} X} G_{1}
$$

By (15), the representative household of type $i$ is liable for a proportion of the overall tax burden $G_{1}$. The proportion is given by the household's share of the overall present value of endowments, with each endowment valued at the competitive equilibrium prices. Although this characterizes VP taxes, it is not a full solution since prices are endogenous. An example with an explicit solution can be constructed by assuming logarithmic utility (identical across households)

$$
u\left(c_{i 1}, c_{i 2}\right)=\log \left(c_{i 1}\right)+\beta \log \left(c_{i 2}\right)
$$

where $0<\beta<1$. Logarithmic utility allows for a closed form solution of the competitive equilibrium given tax policy. Specifically, aggregate capital and prices are independent of taxes and given $b^{21}$

$$
\begin{aligned}
K_{1} & =\frac{\beta \theta}{1+\beta \theta}\left[R_{1} K_{0}+\omega_{1} N_{1}-G_{1}\right] \\
R_{t} & =\theta K_{t-1}^{\theta-1} N_{t}^{1-\theta}, t=1,2 \\
\omega_{t} & =(1-\theta) K_{t-1}^{\theta} N_{t}^{-\theta}, t=1,2
\end{aligned}
$$

The corresponding individual consumption allocations $\left\{c_{i 1}^{*}(\tau, w), c_{i 2}^{*}(\tau, w)\right\}_{i=1}^{2}$, which depend on taxes and endowments, can then be substituted in the weighted average of utilities to solve for Ramsey taxes in the following problem ${ }^{22}$

$$
F_{\lambda}(w)=\max _{\tau} \sum_{i=1}^{2} \lambda_{i} \pi_{i}\left[\log c_{i 1}^{*}(\tau, w)+\beta \log c_{i 2}^{*}(\tau, w)\right]
$$

where $\sum_{i=1}^{2} \lambda_{i} \pi_{i}=1$. Furthermore, the Ramsey maximization problem can be solved analytically to yield a tax policy $\tau^{R A}(w, \lambda)$, consumption allocations $\left\{c_{i 1}^{R A}(w, \lambda), c_{i 2}^{R A}(w, \lambda)\right\}_{i=1}^{2}$ and a social welfare function

$F_{\lambda}(w)=\sum_{i=1}^{2} \lambda_{i} \pi_{i}\left[\log \left(\frac{\lambda_{i}}{1+\beta \theta}\right)+\beta \log \left(\lambda_{i}\right)\right]+\log \left(R_{1} K_{0}+\omega_{1} N_{1}-G_{1}\right)+\beta \log \left(A K_{1}^{\theta} N_{2}^{1-\theta}\right)$

\footnotetext{
${ }^{21}$ Appendix B provides a detailed derivation for this example. Note that leisure is not valued in the utility which implies trivially an inelastic supply of labor, $n_{i t}=X_{i t}$ for all $i, t$. We use $n_{i t}, N_{t}, X_{i t}$ and $X$ interchangeably.

${ }^{22}$ This formulation follows the dual approach where taxes are chosen directly. It is equivalent to the primal approach where allocations are chosen subject to implementability as an equilibrium with taxes.
} 
The closed form solution for $F_{\lambda}$ allows us to explicitly find its partial derivatives and thus write the social contribution of a household of type $i$. Recall that the contribution is given by $\nabla F_{\lambda}(w) \tilde{w}^{i}$. Since the only non-zero elements of $\tilde{w}^{i}$ are the endowments $k_{i 0}, X_{i 1}$ and $X_{i 2}$, we only need to obtain three partial derivatives for each type $i$. Some manipulations and use of equilibrium relations yield

$$
\begin{aligned}
\frac{\partial F_{\lambda}(w)}{\partial\left(\pi_{i} k_{i 0}\right)} & =\frac{1+\beta \theta}{I N C_{1}-G_{1}} R_{1} \\
\frac{\partial F_{\lambda}(w)}{\partial\left(\pi_{i} X_{i 1}\right)} & =\frac{1+\beta \theta}{I N C_{1}-G_{1}} \omega_{1} \\
\frac{\partial F_{\lambda}(w)}{\partial\left(\pi_{i} X_{i 2}\right)} & =\frac{1+\beta \theta}{I N C_{1}-G_{1}} \frac{\omega_{2}}{R_{2}}
\end{aligned}
$$

where $I N C_{1}=R_{1} K_{1}+w_{1} X_{1}$ is aggregate income in period 1 .

The result is consistent with the general result that the gradient is proportional to the equilibrium price vector (see the proof of Proposition 2). Thus the contribution of $\tilde{w}^{i}$ to $F_{\lambda}(w)$ is

$$
C\left(F_{\lambda}, \tilde{w}^{i}, w\right)=\frac{1+\beta \theta}{I N C_{1}-G_{1}} \pi_{i}\left[R_{1} k_{i 0}+\omega_{1} X_{i 1}+\frac{\omega_{2}}{R_{2}} X_{i 2}\right]
$$

The contribution of the individual bundle of goods $x^{i}$ to the individual welfare function $\lambda_{i} \pi_{i} u\left(c_{i 1}, c_{i 2}\right)$ is

$$
C\left(\lambda_{i} \pi_{i} u, x\right)=\lambda_{i} \pi_{i} \frac{1}{c_{i 1}^{R A}(w, \lambda)} c_{i 1}^{R A}(w, \lambda)+\lambda_{i} \pi_{i} \beta \frac{1}{c_{i 2}^{R A}(w, \lambda)} c_{i 2}^{R A}(w, \lambda)
$$

Applying the definition of VP weights and allocations in (2), VP weights are

$$
\lambda_{i}^{V P}=\frac{R_{1} k_{i 0}+\omega_{1} X_{i 1}+\frac{\omega_{2}}{R_{2}} X_{i 2}}{R_{1} K_{0}+\omega_{1} X+\frac{\omega_{2}}{R_{2}} X}
$$

and by $(15) \tau_{i}^{V P}=\lambda_{i}^{V P} G_{1}$.

It is straightforward to obtain the optimal taxes under equal weights $\tau_{i}^{E W}$ by solving the Ramsey problem under the assumption $\lambda_{1}=\lambda_{2}=1$. Equalization of marginal utilities is a necessary condition for that problem and, in the current context, this implies perfect equalization of consumption across individuals. The EW tax simply ensures that initial wealth heterogeneity is wiped out

$$
R_{1} k_{10}-\tau_{1}^{E W}=R_{1} k_{20}-\tau_{2}^{E W}
$$

and hence

$$
\tau_{i}^{E W}=G_{1}+R_{1}\left(k_{i 0}-K_{0}\right)
$$

Comparing (15) to (18), we note that VP taxes are zero when the only motive for taxation is redistribution $\left(G_{1}=0\right)$ whereas EW taxes do the exact opposite and ensure full redistribution. When revenue needs to be raised $\left(G_{1}>0\right)$, both tax systems prescribe tax bills that are linearly increasing in initial capital $k_{i 0}$. However, the VP approach prescribes a flat tax rate on the present value of endowments whereas the EW tax (or subsidy) rates are progressive, again with the objective of full redistribution. 
The VP weights $\lambda_{i}^{V P}$ in this example are linearly increasing in $k_{i 0}$. Although the linearity is due to the assumption of logarithmic utility, it can be shown that VP weights are increasing in $k_{i 0}$ for a general CRRA utility. However, this property is particular to this example and depends crucially on the fact that the gradient of the SWF is proportional to the equilibrium price, meaning that the social value of each good does not depend on the type of individual that brings it. In the following section, we consider environments with distortionary taxation and incomplete markets where this is no longer true. In these environments, the social value of a good is typically higher when it is brought by an agent with low wealth and high marginal utility. As a result, depending on the parameterization and especially on the curvature of the utility function, it is sometimes the case that VP weights are higher for the low $k_{i 0}$ agents.

6.2. Capital versus Labor Income Taxation. We now introduce restrictions on the government's ability to implement desirable allocations by limiting the available tax instruments as is standard in the Ramsey taxation literature. Specifically, we assume the government can raise revenue through flat rate taxes $\tau_{k}$ on capital income and $\tau_{n}$ on labor income. Since we maintain the assumption of no leisure in the utility, both capital and labor income in the first period are inelastic. ${ }^{23}$ To have an interesting problem with distortionary taxation, we now require the government to raise revenue in the second period instead of the first, i.e. we let $G_{1}=0, G_{2} \geq 0$. Relative to the model of the previous section, we replace the individual budget constraints (13) with

$$
\begin{aligned}
c_{i 1}+k_{i 1} & =R_{1} k_{i 0}+\omega_{1} n_{i 1} \\
c_{i 2} & =\left(1-\tau_{k}\right) R_{2} k_{i 1}+\left(1-\tau_{n}\right) \omega_{2} n_{i 2}
\end{aligned}
$$

and the government budget (14) with

$$
G_{2}=\tau_{k} R_{2} K_{1}+\tau_{n} \omega_{2} N_{2}
$$

We continue to assume the logarithmic utility function (16) which implies that labor income taxation is non-distortionary. Therefore, the efficient tax scheme, in the sense of maximizing aggregate production $Y_{2}$, is to tax only labor income. However, the choice of welfare weights will determine societal preference for equality and that can conflict with a pure efficiency objective. In what follows, we compare the prescribed optimal tax schemes under VP and EW weights.

Since we no longer have an analytical solution available, we obtain allocations, taxes and VP weights numerically based on the following parameter choices. The discount factor is $\beta=0.9$ and the capital income share in total income is set to $\theta=0.4$. We assume the two types of agents are of equal measure, $\pi_{1}=0.5$, and normalize time endowments to one, $X_{i t}=1$. Aggregate initial capital $K_{0}$ is also normalized to one, with type 1 agents receiving a fraction $\phi\left(=\frac{\pi_{1} k_{10}}{K_{0}}\right)$ and type 2 agents receiving a fraction $1-\phi$. When $\phi=\pi_{1}(=0.5)$ there is no heterogeneity. As $\phi$ is decreased towards zero, type 1 agents become progressively more

\footnotetext{
${ }^{23}$ We do not include the analysis of the endogenous labor supply case because it does not add new insights about VP versus EW welfare functions.
} 
wealth-poor relative to type 2 agents. By varying $\phi$ we can investigate the effects of different levels of wealth inequality on optimal taxation under VP weights and under EW weights.

Consider first the case $G_{2}=0$. In this case, the only motive for taxation is redistribution. Capital and labor tax rates are shown in Figure 1 as a function of initial wealth inequality $\phi{ }^{24}$. When agents are identical $(\phi=0.5)$ all taxes are zero regardless of the choice of weights. Introducing wealth inequality $(\phi<0.5)$ does not change the zero tax prescription under VP weights because these mute the redistribution motive. ${ }^{25}$ Equal weights, on the other hand, introduce a strong preference for redistribution. As a result, as wealth inequality increases towards $\phi=0$ the EW approach prescribes increasing tax rates on capital income and subsidy rates on labor income. This is because second period capital income is higher for the type that has higher initial wealth whereas second period labor income is the same for all types. Thus, this scheme redistributes from the higher wealth types to the lower wealth types. Because the government is restricted to using distortionary capital income taxes, it has to trade-off the redistribution motive with efficiency considerations. As a result, redistribution is not perfect, but the capital tax clearly grows with the level of inequality.

Figure 1: Complete Markets, $G_{2}=0$

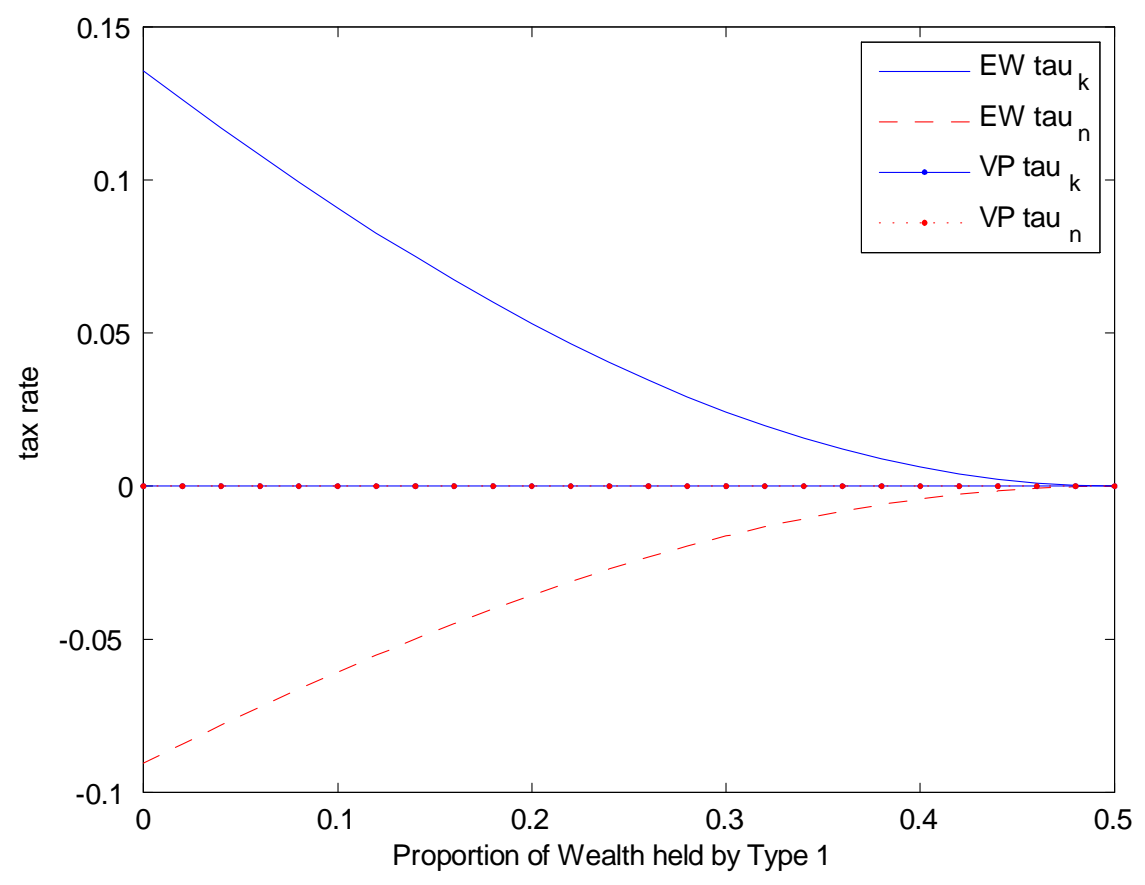

Consider now the case shown in Figure 2 in which the government needs to raise revenue, $G_{2}>0$. The efficient way to raise revenue is by taxing labor income and not capital income

\footnotetext{
${ }^{24}$ We only plot the figures for levels of inequality between 0 and 0.5 , since the picture is symmetric for inequality levels between 0.5 and 1 .

${ }^{25}$ The fact that $\tau_{k}=\tau_{n}=0$ is a VP tax policy can be shown by following arguments similar to those in Proposition 2.
} 
and this is exactly the optimal tax scheme at $\phi=0.5$ where agents are identical. ${ }^{26}$ Under the EW approach, the higher the level of inequality, the stronger the incentive to tax capital and lower taxes on labor income. If initial wealth inequality is large enough, this approach can end up prescribing higher taxation for capital than for labor, purely for redistribution purposes. This does not happen under VP tax rates, which are always higher for labor income. However, VP taxes are not invariant to inequality levels. To understand this, suppose that capital taxes remained at zero even when the overwhelming majority of initial wealth is held by one of the types. That would imply that the fraction of the present value of endowments paid in taxes to the government would be much higher for the wealth poor than the wealth rich. In other words, using only labor taxes to raise revenues would imply (reverse) redistribution. Instead, to respect the no redistribution principle, the labor tax rate has to fall and the capital tax rate has to rise as inequality increases. Still, even for large amount of inequality labor taxes remain above capital taxes.

To summarize, for both VP and EW weights, distributional concerns prevent the tax scheme from being fully efficient, but the EW approach requires a larger deviation because it embeds a strong preference for equality.

Figure 2: Complete Markets, $G_{2}>0$

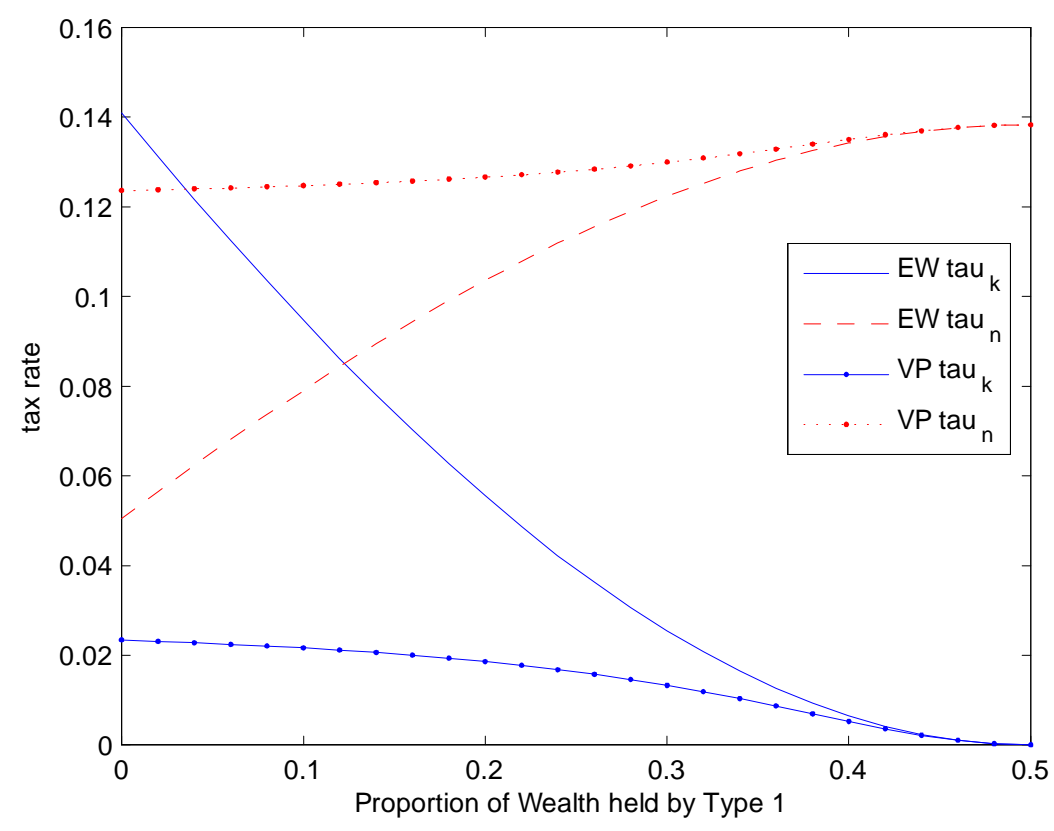

In addition to raising revenue and achieving redistribution, another motive for the use of taxation by the government can be provided by the lack of insurance markets. In the presence of idiosyncratic risk, and in the absence of private markets that can insure against such risk, tax policy could play a role in providing such insurance. In our last modification,

\footnotetext{
${ }^{26}$ If agents derived utility from leisure, labor supply would be elastic and this would not be true necessarily. However, we do not focus here on the level of capital versus labor taxes. Instead we are interested in the effect of an increase in inequality. The effect on capital and labor income taxes described in what follows applies equally to the case of endogenous labor supply.
} 
we introduce uninsurable idiosyncratic risk by replacing the wage income in the second period with $e \omega_{2}$, where $\omega_{2}$ is still the wage rate and $e$ is a random variable capturing labor efficiency. We assume no aggregate uncertainty to stay as close as possible to a standard Aiyagari (1994, 1995) framework. In particular, in every state of nature and for each household type $i$ there is a proportion $\pi^{h}$ of households that receives the high efficiency shock and a proportion $\left(1-\pi^{h}\right)$ of households that receives the low efficiency shock. Each state of nature is equally likely and, as a result each individual faces the following distribution of $e$

$$
e=\left\{\begin{array}{c}
e^{h} \text { with prob. } \pi^{h} \\
e^{l} \text { with prob. } 1-\pi^{h}
\end{array}\right\}
$$

The average level of efficiency is normalized to one, i.e. $\pi^{h} e^{h}+\left(1-\pi^{h}\right) e^{l}=1$ and we choose, $\pi^{h}=0.5, e^{l}=0.8$ and $e^{h}=1.2$ for the numerical computations.

There is a sense in which insurance and redistribution are two sides of the same coin. As pointed out by Davila et. al (2012), what we call insurance ex ante (before the resolution of uncertainty) is redistribution from an ex post point of view. Redistribution, however, can have a wider scope, since it is about undoing inequality regardless of its causes. To put it simply, it is hard to argue that all of inequality is due to chance. The stylized model considered here does not take a stand on the origins of initial wealth inequality. On the other hand, it is clear that second period labor income inequality is purely down to chance. This arrangement, stylized as it is, provides a nice example of the contrasting motives of insurance and redistribution. Given the government's available instruments $\tau_{n}$ and $\tau_{k}$, the way to provide insurance is to shift the burden of taxation to labor income. This has the effect of reducing the risky part of income and increasing the safe part. Interestingly, this is exactly the opposite of what redistribution would require here. Redistribution (of initial wealth inequality) is achieved by shifting the burden of taxation to capital income. We view the VP weights as a way to disentangle and isolate these two motives.

Figure 3 shows the results for this case. When $\phi=0.5$, there is no redistribution motive. Whereas with complete markets this implied that all revenue was raised through labor taxes, when markets are incomplete taxes can also be used for the provision of insurance. The tax scheme that can (partly) achieve this calls for a tax on risky labor income and a subsidy on capital income. As wealth inequality increases, the VP tax scheme needs to be adjusted to ensure the value preserving principle still holds. This requires a reduction in the labor tax and a reduction in the capital subsidy, since otherwise there would be a redistribution of value towards capital owners. The EW scheme moves in the same direction but much stronger in an attempt to redistribute towards the wealth poor. Similarly to the case of complete markets, only moderate levels of inequality are enough to reverse the prescription and call for taxing capital more than labor. The redistribution motive under EW is quite strong. The conclusion is that insurance and redistribution can work in opposite directions and the VP approach can provide a method to abstract from the redistribution effect and shed light on the pure insurance effect. 
Fig. 3: Incomplete Markets, Capital/Labor, $G_{2}>0$

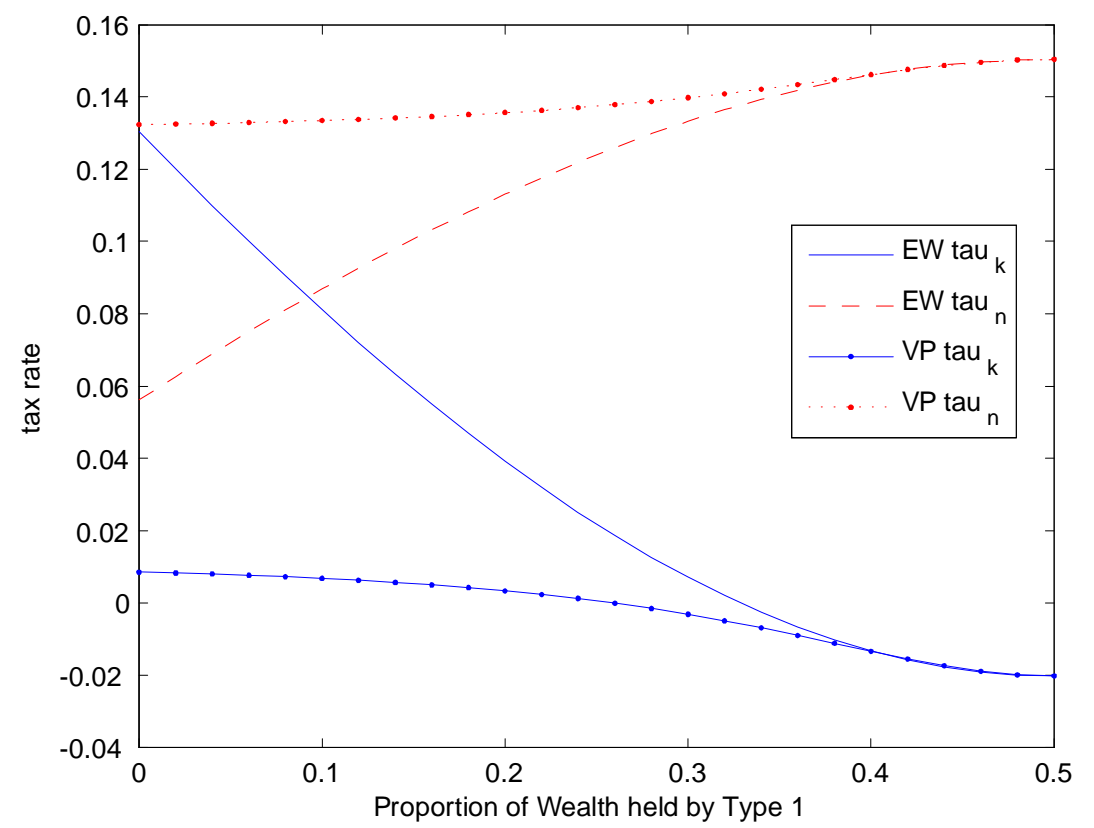

\section{Discussion of Alternative Approaches}

We have used a specific principle, the value-preserving principle, to determine endogenously the welfare weights for social optimization problems. The VP principle postulates that every individual should be rewarded in proportion to his or her contribution to welfare. To calculate this contribution we used a mechanism based on the axiomatization of Section 4. In this section, we discuss alternative contribution mechanisms as well as alternative principles that can be used to determine social welfare weights.

7.1. Alternative Contribution Mechanisms. Our approach is closely related to Mankiw's (2010) "just deserts" theory, which calls for individuals receiving compensation that is congruent with their contribution and for the contribution to be measured according to marginal productivity theory. Mankiw (2010) argues that individuals would exercise the right to leave society and live on their own if they felt their contributions were insufficiently rewarded. This ensures that, under standard assumptions, allocations will be in the core and, for sufficiently large economies, they will be sufficiently close to a Walrasian competitive equilibrium, in which the factors of production are paid their marginal products. However, as Weinstein (2011) argues, neither the assumptions that satisfy the marginal productivity compensation principle nor the convergence to a competitive equilibrium need hold in reality.

Our contribution mechanism is reminiscent of Mankiw's appeal to marginalism because it assigns value to goods according to their marginal effects on social welfare. However, it is not directly subject to some of the criticisms aimed at marginal productivity theory because it does not necessarily rely on the economy being frictionless and delivering market prices equal to marginal products. Indeed, our approach is developed so that it can be used in 
economies with incomplete markets, externalities or other frictions.

A more general criticism of marginalism, that Weinstein (2011) also alludes to, is that marginal product accounting can help to determine the best way to use additional resources, but it does not really capture the overall contribution of a finite amount of resources. Partly based on this, Weinstein argues that the marginal value allocation "may not match a reasonable notion of fairness". Instead, he proposes to use the classic idea of the Shapley value, which is based on some basic principles of fairness (see Shapley (1953)). ${ }^{27}$ In what follows, we discuss the underlying assumptions as well as the implications of an alternative approach that eschews marginalism and connects more closely to the Shapley value. Interestingly, we argue that, with utilities widely used in macroeconomic applications, this seemingly very different approach yields the exact same result as our contribution mechanism.

Starting from our approach, recall that the mapping $C(F, w)=\alpha \nabla F(w)$, for $\alpha \in \mathbb{R}_{+}^{m}$, $w \in \mathbb{R}_{+}^{m} \backslash\{0\}$ and $F: \mathbb{R}_{+}^{m} \rightarrow \mathbb{R}$, is the unique mechanism that satisfies our three axioms of rescaling, separability and continuity. This mapping has several properties: (i) additivity, namely, $C(F+G, w)=C(F, w)+C(G, w)$ for all $F, G \in \mathcal{F}^{m}$; (ii) consistency, namely, if $F: \mathbb{R}_{+}^{m} \rightarrow \mathbb{R}$ and $G: \mathbb{R} \rightarrow \mathbb{R}$ such that $F\left(x_{1}, \ldots, x_{m}\right)=G\left(\sum_{i=1}^{m} x_{i}\right)$, then $C_{j}(F, w)=$ $C\left(G, \sum_{i=1}^{m} w_{i}\right)$; (iii) positivity, namely for $w \in \mathbb{R}_{+}^{m} \backslash\{0\}$, if $F-G$ is non decreasing in the box $\Pi_{j=1}^{m}\left[0, w_{j}\right]$ then $C(F, w) \geq C(G, w) F, G \in \mathcal{F}^{m}$; (iv) weak rescaling, namely, if $F, G \in \mathcal{F}^{m}$ and $r \in \mathbb{R}_{++}^{m}$ such that $G\left(x_{1}, \ldots, x_{m}\right)=F\left(r_{1} x_{1}, \ldots, r_{m} x_{m}\right)$, then $C_{j}(G, w)=r_{j} C_{j}(F, r * w)$ for $j=1, \ldots, m$, where $r * w=\left(r_{1} w_{1}, \ldots, r_{m} w_{m}\right)$.

Taking these four properties as axioms, Samet and Tauman (1982) show in a cost allocation context that $C(\cdot, \cdot)$ is a per unit contribution mechanism satisfying these four axioms if and only if there exists a unique non-negative measure $\mu$ on $([0,1], \beta)$ ( $\beta$ is the set of all Borel subsets of $[0,1])$ such that for each positive integer $m$, for each $F \in \widetilde{\mathcal{F}}^{m}$ and for each $w \in \mathbb{R}_{+}^{m} \backslash\{0\}$ and $1 \leq j \leq m$

$$
C_{j}(F, w)=\int_{0}^{1} \frac{\partial F}{\partial x_{j}}(t w) d \mu(t)
$$

where $\widetilde{\mathcal{F}}^{m}$ is the set of all continuously differentiable functions $F$ on $\mathbb{R}_{+}^{m}$ such that $F(0)=0$.

The result asserts that any non negative measure $\mu$ on $[0,1]$ (atomic or non atomic) defines by (21) a contribution mechanism that satisfies the four axioms and vice versa, any contribution mechanism that satisfies the four axioms must be given by (21) for some non negative measure. In other words, equation (21) defines a one to one mapping from all the nonnegative measures on $([0,1], \beta)$ onto the set of all contribution mechanisms satisfying the four axioms.

The axiomatic approach above does not specify what measure $\mu$ should be used. One particular case is the atomic measure which is fully concentrated at $t=1$, with $\mu([0,1))=0$ and $\mu(1)=1$, corresponding to our marginal contribution mechanism. Samet and Tauman (1982) also show that one can uniquely characterize this particular mechanism with the

\footnotetext{
${ }^{27}$ In a game theoretical context, the Shapley value of an individual measures his or her contribution to the welfare of society (the worth of the grand coalition) via his average marginal contribution to a random coalition of players.
} 
previous axioms but with a stronger positivity requirement ${ }^{28}$. Instead, we use a different axiomatic approach that disposes of the additivity axiom and of the restriction that $F(0)=0$, which are less appealing in the context of welfare functions ${ }^{29}$.

It can also be shown (see Mirmann and Tauman (1982)) that if we add to the previous four axioms the requirement that the contributions add up to the total welfare ${ }^{30}$, the unique measure $\mu$ satisfying the five axioms is the Lebesgue measure on $[0,1]$, namely, for $j=1, \ldots, m$

$$
C_{j}(F, w)=\int_{0}^{1} \frac{\partial F}{\partial x_{j}}(t w) d t
$$

This measure, which is again a particular case of the family of contribution mechanisms in (21), is the uniform average of the marginal contribution to welfare of commodity $j$ along the diagonal $[0, w]$. Mirmann and Tauman (1982) show that given a pair $(F, w)$ one can define a non atomic game (with a continuum of players) by treating every commodity $j$ of size $w_{j}$ as a set of infinitesimal players, each represented by a point in the interval $\left[0, w_{j}\right]$. The value of this non atomic game, which is an extension of the Shapley value from a finite number of players to the continuum (see Aumann and Shapley (1974)) is closely related to (22). Whereas the axioms in Mirmann and Tauman (1982) extend the ones of Aumann and Shapley (1974) to an economic context, the fairness inherent in the axioms is less apparent, since the game described above is between commodities rather than individuals. Instead, one could define a finite coalitional game $v_{\lambda}(S)$, for a given a set of weights $\left(\lambda_{i}\right)_{i \in I}$ and any coalition of players $S \subseteq I$, as the maximum welfare level that individuals in $S$ could achieve on their own. The contribution of an individual to the welfare of society (the worth of the grand coalition) can then be computed using the Shapley (1953) value, which is equal to his or her average marginal contribution to a random coalition of players. In turn, the VP weights would equate the ratio of social contributions to individual weighted utility across agents. Since the players are individuals and not commodities, the fairness with this approach would be more apparent.

In sum, we have provided a family of mechanisms in (21) that can potentially be used to calculate the contribution of agents to welfare via the weighted average marginal value of the initial endowments. In turn, this contribution can be used to compute the social welfare weights using our VP principle. Our marginal value approach, which is a particular case, is the only one for which the VP weights generate Walrasian equilibrium allocations in the absence of frictions. However, there is a case in which all the mechanisms coincide: the

\footnotetext{
${ }^{28}$ The stronger positivity axiom states that if $(F-G)(x)$ is non decreasing for $x \leq w$ in a neighborhood of $w \in \mathbb{R}_{+}^{m} \backslash\{0\}$ then $C_{j}(F, w) \geq C_{j}(G, w)$, for $1 \leq j \leq m$ and all $F, G \in \mathcal{F}^{m}$. This axiom requires that the per unit contribution to the SWF $F$ of the $j^{\text {th }}$ good is at least as high as its contribution to the SWF $G$ whenever $F-G$ is non decreasing in a neighborhood of $w$

${ }^{29}$ To avoid the restriction that $F(0)=0$, one could also give up the axiomatic approach altogether and calculate the contribution of a good to any welfare function using any measure that is concentrated at any particular point between 0 and 1 .

${ }^{30}$ Formally, this axiom would require that for every $F \in \mathcal{F}^{m}$ and every $w \in \mathbb{R}_{+}^{m}$
}

$$
\sum_{j=1}^{m} w_{j} C_{j}(F, w)=F(w) .
$$


case of homogeneous utilities. More precisely, under the same assumptions of Proposition 1 , it can be shown that if all utilities $\left(u_{i}\right)_{i \in I}$ are homogeneous of degree $r$ for some $r>0$, an allocation is VP for any contribution mechanism satisfying (21) iff it is a Walrasian (competitive) equilibrium with respect to the initial endowments $w$.

7.2. Alternative Principles. The previous section discusses alternative contribution mechanisms that can be used in conjunction with the VP principle to determine social welfare weights. Here, we take our contribution mechanism as given and discuss how it can be used in the application of principles other than the VP principle. In particular, we look at two popular alternatives to utilitarianism, namely the equal sacrifice principle and the Rawlsian principle.

Consider first the equal sacrifice principle. To see how this principle could be used to determine social welfare weights using our approach, note that one would first have to take a stand about the starting point from which each individual's sacrifice is calculated. Suppose we assume that this starting point is the first best allocation. ${ }^{31}$ The equal sacrifice condition can then be stated as follows:

$$
\frac{C\left(\lambda_{i} u_{i}, x_{\lambda}^{i}\right)}{C\left(\lambda_{i}^{N} u_{i}, x_{N}^{i}\right)}=\frac{C\left(\lambda_{h} u_{h}, x_{\lambda}^{h}\right)}{C\left(\lambda_{h}^{N} u_{h}, x_{N}^{h}\right)} \text { for all } i, h \in I
$$

where $\lambda^{N}=\left(\lambda_{i}^{N}\right)_{i \in I}$ are the Negishi weights corresponding to the first best allocation, which we denote by $x_{N} \in \mathbb{R}_{+}^{L I}$, and $x_{\lambda} \in \mathbb{R}_{+}^{L I}$ is the final bundle in the allocation with taxes for a given a set of weights $\lambda=\left(\lambda_{i}\right)_{i \in I}$. Condition (23) states that the ratio of the private value of the tax allocation relative to the private value of the first best allocation has to equalize across agents under the equal sacrifice weights. Here, it is important to note that this formulation uses our contribution mechanism to compute the private value to the agent of different allocations and the resulting allocations are invariant under non-uniform affine transformations of utilities. In contrast, this desirable property will not be satisfied if one uses instead the ratio of weighted utility levels $\lambda_{i} u_{i}$ in the tax and first best allocations to compute the welfare weights, as is common in the literature.

Our contribution mechanism can also be used to determine social welfare weights that would correspond to a Rawlsian principle. These weights would be determined by:

$$
\arg \max _{\lambda} \min _{i}\left\{C\left(\lambda_{i} u_{i}, x_{\lambda}^{i}\right)\right\}
$$

where $x_{\lambda}^{i}$ is the allocation with taxes for a given set of weights $\lambda=\left(\lambda_{i}\right)_{i \in I}$ and $C\left(\lambda_{i} u_{i}, x_{\lambda}^{i}\right)$ is the private value to the agent of this allocation. Essentially the Rawlsian weights would maximize the private value to the individual with the lowest payoff. As with the previous mechanism, the allocations resulting from this problem are invariant under non-uniform affine transformations of utilities. However, if we use the weighted utility levels $\lambda_{i} u_{i}$ instead of the contribution to welfare $C\left(\lambda_{i} u_{i}, x_{\lambda}^{i}\right)$, this property will fail, since the allocations will not be invariant under transformations that add different constants to different utilities.

\footnotetext{
${ }^{31}$ Note that, although common in the literature, this choice is arbitrary and is made for illustrative purposes. One of the benefits of our VP principle relative to the equal sacrficie principle is that it naturally avoids having to make this choice of a reference economy altogether.
} 


\section{Conclusion}

This paper provides an axiomatic approach to determine the contribution of a bundle of goods (the value of a bundle) to any welfare function, regardless of whether it is a social or an individual welfare function. We then postulate a value preserving principle which consists in equating across agents the ratio of the value of the initial bundle of goods to social welfare to the value of the final bundle of goods to private welfare. This principle is used to choose amongst different welfare weights or among different constrained efficient allocations. We show that these weights, which we refer to as value preserving weights, can be thought of as an extension of the classic Negishi weights to non Arrow-Debreu economies. In an example of optimal taxation, we point out the usefulness of our approach in disentangling insurance and redistribution motives for taxation. This can help clarify existing analyses of optimal taxation of capital versus labor income.

Our approach could be used in other interesting applications. The first is the study of constrained efficient allocations under incomplete markets. Using an equal-weights approach, Davila, Hong, Krusell and Rios-Rull (2012) investigate the constrained efficient level of aggregate capital under incomplete markets. In particular, they investigate whether the equilibrium level of capital is too high or too low. For the most commonly used specification of risk, they find that the competitive equilibrium level of aggregate capital is too low. The extent to which this finding relies on the equal-weights assumption is not obvious. To put it differently, it remains unclear whether the constrained efficient level of capital is higher because this provides better insurance or more redistribution and the VP approach can shed some light on this question. Another potential application is the endogenous determination of the objective for the firm when markets are incomplete and shareholders disagree. In such a setting, one could aggregate the preferences of shareholders by maximizing a weighted average of their utilities, using value preserving weights that equate across shareholders the ratio of the contribution of the initial investment to the firm value and the contribution of the final allocation to private welfare.

\section{Appendix A: Proofs}

\section{Proof of Theorem 1.}

It is straightforward to verify that the per unit contribution mechanism $\widehat{C}_{j}(F, w)=$ $\alpha \frac{\partial F}{\partial x_{j}}(w)$ for $1 \leq j \leq m$ defined for every $F \in \mathcal{F}^{m}$ and $w \in \mathbb{R}_{+}^{m} \backslash\{0\}$ satisfies the three axioms. The proof of the other direction is less obvious. Suppose that $\widehat{C}_{j}(\cdot, \cdot)$ is a per unit contribution mechanism that satisfies the three axioms.

We first prove the theorem for linear welfare functions of the form $F(x)=\sum_{j=1}^{m} b_{j} x_{j}$. Namely, we have to show that $\widehat{C}_{j}(F, w)=\alpha b_{j}, 1 \leq j \leq m$ for $\alpha=\widehat{C}(I, 1)$, where $I(x)=x$ for all $x \in \mathbb{R}$. We first show that $\widehat{C}(I, 1)=\widehat{C}(I, a)$ for all $a \in \mathbb{R}_{++}$. Let $F(x)=I(a x)$. By the rescaling axiom for the units of the commodity, $\widehat{C}(F, 1)=a \widehat{C}(I, a)$. On the other hand, $F=a I$, hence $\widehat{C}(F, 1)=\widehat{C}(a I, 1)=a \widehat{C}(I, 1)$ (by the rescaling axiom). These imply $\widehat{C}(I, a)=\widehat{C}(I, 1)$. Next define $F^{j}\left(x_{j}\right)=b_{j} x_{j}$. Then $F=\sum_{j=1}^{m} F^{j}\left(x_{j}\right)$. By separability 
and the rescaling of welfare units,

$$
\widehat{C}_{j}(F, w)=\widehat{C}\left(F^{j}, w_{j}\right)=\widehat{C}\left(b_{j} I, w_{j}\right)=b_{j} \widehat{C}\left(I, w_{j}\right)
$$

Suppose first that $w_{j}>0$. Then

$$
\widehat{C}_{j}(F, w)=b_{j} \widehat{C}(I, 1)=b_{j} \alpha .
$$

Next suppose that $w_{j}=0$. By the continuity axiom, for $\epsilon>0$ there exists $\eta>0$ sufficiently small s.t. $|\widehat{C}(I, 0)-\widehat{C}(I, \eta)|<\epsilon$. But $\widehat{C}(I, \eta)=\widehat{C}(I, 1)=\alpha$. Hence $|\widehat{C}(I, 0)-\alpha|<$ $\epsilon$. Since this is true for any $\epsilon>0, \widehat{C}(I, 0)=\alpha$ and $\widehat{C}_{j}(F, w)=b_{j} \alpha$.

Next we prove the general case. Let $F \in \mathcal{F}^{m}$ and $w \in \mathbb{R}_{+}^{m} \backslash\{0\}$. Since $F$ is continuously differentiable on $\mathbb{R}_{+}^{m} \backslash\{0\}$

$$
F(x)=\nabla F(w) x+F(w)-w \nabla F(w)+o(w-x)
$$

Let $\epsilon>0$. By the continuity axiom there exists $\eta_{1}>0$ and $\delta>0$ s.t. for all $G \in \mathcal{F}^{m}$ with $\|F-G\|_{X\left(w, \eta_{1}\right)}^{1}<\delta$

$$
\left|\widehat{C}_{j}(F, w)-\widehat{C}_{j}(G, w)\right|<\epsilon, 1 \leq j \leq m
$$

Let $G(x)=\nabla F(w) x+c$, where $c=F(w)-w \nabla F(w)$. For the above $\delta$ there exists $\eta_{2}>0$ s.t. $\|o(w-x)\|_{X\left(w, \eta_{2}\right)}^{1}<\delta$.

Let $\eta=\min \left(\eta_{1}, \eta_{2}\right)$. By $(24)$ and by the definition of the $C^{1}$ norm

$$
\|F-G\|_{X(w, \eta)}^{1} \leq\|F-G\|_{X\left(w, \eta_{1}\right)}^{1}<\delta
$$

Therefore,

$$
\left|\widehat{C}_{j}(F, w)-\widehat{C}_{j}(G, w)\right|<\epsilon
$$

By axiom 1,

$$
\widehat{C}_{j}(G, w)=\widehat{C}_{j}(\nabla F(w) x, w)
$$

Since $\nabla F(w) x$ is linear

$$
\widehat{C}_{j}(G, w)=\frac{\partial F}{\partial x_{j}}(w) \alpha
$$

Substituting this into (25)

$$
\left|\widehat{C}_{j}(F, w)-\frac{\partial F}{\partial x_{j}}(w) \alpha\right|<\epsilon
$$

Since the last inequality holds for all $\epsilon>0$, we conclude that $\widehat{C}_{j}(F, w)=\frac{\partial F}{\partial x_{j}}(w) \alpha$ and the proof of the theorem is complete.

Proof of Proposition 1. We start by showing that if $\lambda$ is a Negishi weight then it is value preserving. Let $\lambda \in \Delta^{I}$ be a Negishi weight. Using (4), (5) and applying the envelope theorem it is straightforward to show (see for example Mas-Colell et al (1995)) that

$$
\nabla F_{\lambda}(w)=\frac{p}{K}=\lambda_{i} \nabla u_{i}\left(x_{\lambda}^{i}(w)\right)
$$


where $K \equiv \sum_{i=1}^{I} \frac{1}{\mu_{i}}$ is a constant. ${ }^{32}$ Multiplying by $x_{\lambda}^{i}(w)$ we obtain

$$
\lambda_{i} \nabla u_{i}\left(x_{\lambda}^{i}(w)\right) \cdot x_{\lambda}^{i}(w)=\nabla F_{\lambda}(w) \cdot x_{\lambda}^{i}(w)=\frac{p}{K} \cdot x_{\lambda}^{i}(w)
$$

Since the budget constraint in the competitive equilibrium problem must hold,

$$
\begin{aligned}
p \cdot x_{\lambda}^{i}(w) & =p \cdot\left(w^{i}+\sum_{j=1}^{J} \theta_{j}^{i} z_{\lambda}^{j}(w)\right) \\
& =p \cdot w^{i}
\end{aligned}
$$

where the last equality follows from the fact that the $f_{j}$ are homogeneous of degree 1 . We have thus shown that

$$
\lambda_{i} \nabla u_{i}\left(x_{\lambda}^{i}(w)\right) \cdot x_{\lambda}^{i}(w)=\nabla F_{\lambda}(w) \cdot w^{i}
$$

which by Theorem 1 implies

$$
C\left(\lambda_{i} u_{i}, x_{\lambda}^{i}(w)\right)=C\left(F_{\lambda}, \tilde{w}^{i}, w\right)
$$

and the Negishi weight $\lambda$ is value preserving.

We now prove the other direction, namely, we show that if $\lambda$ is a VP weight then it is a Negishi weight for the initial endowments $w$. Let $\lambda \in \Delta^{I}$ be a value preserving weight and let $(\bar{x}, \bar{z})$ be the corresponding VP allocations so that

$$
\nabla F_{\lambda}(w) \cdot w^{i}=c \lambda_{i} \nabla u_{i}\left(\bar{x}^{i}\right) \cdot \bar{x}^{i}
$$

for some $c \in \mathbb{R}_{+}$. Since $(\bar{x}, \bar{z})$ is a Pareto optimal allocation, by the second welfare theorem there exists some $\bar{p} \in \mathbb{R}_{+}^{L}$ such that for every $i, \bar{x}^{i}$ maximizes $u_{i}\left(x^{i}\right)$ over $\left\{x^{i} \mid \bar{p} \cdot x^{i} \leq \bar{p} \cdot \bar{x}^{i}\right\}$ and $\bar{z}^{j}$ maximizes $\bar{p} \cdot z^{j}$ over $f_{j}\left(z^{j}\right)=0$. Assuming an interior solution, there exists $\alpha>0$ such that for every $i \in I$

$$
\alpha \bar{p}=\lambda_{i} \nabla u_{i}\left(\bar{x}^{i}\right)=\nabla F_{\lambda}(w)
$$

By (26) $\bar{p} \cdot w^{i}=c \bar{p} \cdot \bar{x}^{i}, i \in I$. Since $\bar{p} \cdot \bar{z}^{j}=0$ and $\sum_{i \in I} \bar{x}^{i}=\sum_{i=1}^{I} w^{i}+\sum_{j \in J} \bar{z}^{j}$, we have that $c=1$. Hence, for all $i \in I$

$$
\bar{p} \cdot \bar{x}^{i}=\bar{p} \cdot w^{i}
$$

and $\bar{x}^{i}$ maximizes $u_{i}\left(x^{i}\right)$ over $\left\{x^{i} \mid \bar{p} \cdot x^{i} \leq \bar{p} \cdot w^{i}\right\}$, implying that $\lambda$ is a Negishi weight

Proof of Proposition 2: Let $Z_{j}=\left\{z^{j} \mid f_{j}\left(z^{j}\right) \leq 0\right\}, B(G)=\left\{\tau=\left(\tau^{i}\right)_{i \in I} \mid \tau^{i} \in \mathbb{R}_{+}^{L}, \sum_{i \in I} \tau^{i}=G\right\}$ and $w=\left(w^{1}, \ldots, w^{I}\right) \in \mathbb{R}_{+}^{L I}$. Let the set of feasible allocations with respect to $W-G$ be

$$
\bar{L}(G)=\left\{(x, z) \mid \sum_{i \in I} x^{i}=W-G+\sum_{j \in J} z^{j}, f_{j}\left(z^{j}\right) \leq 0, j=1, \ldots, J\right\}
$$

\footnotetext{
${ }^{32}$ Note that, in this case, the value $F_{\lambda}$ depends on the aggregate endowment $W=\left(W_{1}, \ldots, W_{L}\right) \equiv \sum_{i=1}^{I} w^{i} \in$ $\mathbb{R}_{++}^{L}$ only and not on the whole distribution $w$. The implication is that $\frac{\partial F_{\lambda}}{\partial w_{l}^{i}}=\frac{\partial F_{\lambda}}{\partial w_{l}^{h}}=\frac{\partial F_{\lambda}}{\partial W_{l}}$ for all $i, h \in I$ and the notation $\nabla F_{\lambda}(w)$ should be interpreted to mean $\left(\frac{\partial F_{\lambda}}{\partial W_{1}}, \ldots, \frac{\partial F_{\lambda}}{\partial W_{L}}\right)$
} 
and recall that the set of allocations implementable as a competitive equilibrium with lump sum taxes is

$$
\widehat{L}(G)=\left\{(\widehat{x}, \widehat{z}) \mid \text { there exist } \widehat{\tau} \in \mathbb{R}^{I L} \text { and } \widehat{p} \in \mathbb{R}_{+}^{L} \text { s.t. }(\widehat{x}, \widehat{z}, \widehat{\tau}, \widehat{p}) \text { satisfies (6)-(10) }\right\}
$$

For any $\lambda \in \Delta_{I}$, let

$$
\begin{aligned}
& \bar{F}_{\lambda}(w)=\max _{(x, z)} \sum_{i \in I} \lambda_{i} u_{i}\left(x^{i}\right) \text { s.t. }(x, z) \in \bar{L}(G) \\
& \widehat{F}_{\lambda}(w)=\max _{(x, z)} \sum_{i \in I} \lambda_{i} u_{i}\left(x^{i}\right) \text { s.t. }(x, z) \in \widehat{L}(G)
\end{aligned}
$$

Lemma 6. $(x, z)$ is a maximizer of $\widehat{F}_{\lambda}(w)$ iff it is a maximizer of $\bar{F}_{\lambda}(w)$ and hence $\bar{F}_{\lambda}(w)=\widehat{F}_{\lambda}(w)$.

Proof: Since $\widehat{L}(G) \subseteq \bar{L}(G)$ it follows that $\widehat{F}_{\lambda}(w) \leq \bar{F}_{\lambda}(w)$. To prove the converse inequality, let $(\bar{x}, \bar{z})$ be a maximizer of $\bar{F}_{\lambda}(w)$. Since $(\bar{x}, \bar{z})$ is a Pareto optimal allocation with respect to the initial resources $\sum_{i \in I} w^{i}-G$, by the second welfare theorem, there exists a supporting price $\bar{p} \in \mathbb{R}_{+}^{L}$ s.t. for every $i \in I, \bar{x}^{i}$ maximizes $u_{i}\left(x^{i}\right)$ over $\left\{x^{i} \in \mathbb{R}_{+}^{L} \mid \bar{p} x^{i} \leq \bar{p} \bar{x}^{i}\right\}$ and for every $j \in J, \bar{z}^{j}$ maximizes $\bar{p} z^{j}$ over $z^{j}$ s.t. $f_{j}\left(z^{j}\right) \leq 0$. Now let

$$
\bar{\tau}^{i}=w^{i}-\bar{x}^{i}+\sum_{j \in J} \theta_{j}^{i} \bar{z}^{j}, i \in I
$$

where $\theta_{j} \in \mathbb{R}_{+}^{I}$ are the initial shares of agent $i$ in firm $j$ so that $\sum_{i=1}^{I} \theta_{j}^{i}=1$ for all $j$. Then, by $(6)$,

$$
\sum_{i \in I} \bar{\tau}^{i}=\sum_{i \in I} w^{i}-\sum_{i \in I} \bar{x}^{i}+\sum_{i \in I} \sum_{j \in J} \theta_{j}^{i} \bar{z}^{j}=G
$$

Also, since $\bar{p} \bar{z}^{j}=0$ for all $j \in J$ by the homotheticity of technologies, we know $\bar{p} \sum_{j \in J} \bar{z}^{j}=0$ and therefore

$$
\bar{p} \bar{\tau}^{i}=\bar{p}\left(w^{i}-\bar{x}^{i}\right)
$$

Hence, $\bar{x}^{i}$ maximizes $u_{i}\left(x^{i}\right)$ over $\left\{x^{i} \in \mathbb{R}_{+}^{I} \mid \bar{p} x^{i} \leq \bar{p}\left(w^{i}-\bar{\tau}^{i}\right)\right\}$. Thus, $(\bar{x}, \bar{z}, \bar{\tau}, \bar{p})$ satisfies the five conditions $(6)-(10)$ and $(\bar{x}, \bar{z}) \in \bar{L}(G)$. This implies that

$$
\bar{F}_{\lambda}(w)=\sum_{i \in I} \lambda_{i} u_{i}\left(\bar{x}^{i}\right) \leq \widehat{F}_{\lambda}(w)
$$

Since $\bar{F}_{\lambda}(w) \geq \widehat{F}_{\lambda}(w)$ also holds, we have that $\bar{F}_{\lambda}(w)=\widehat{F}_{\lambda}(w)$ and $(\bar{x}, \bar{z})$ is also a maximizer of $\widehat{F}_{\lambda}(w)$

Suppose next that $\lambda$ is a VP weight. Then,

$$
\frac{\nabla \widehat{F}_{\lambda}(w) \tilde{w}^{i}}{\nabla \widehat{F}_{\lambda}(w) \tilde{w}^{h}}=\frac{\lambda_{i} \nabla u_{i}\left(\widehat{x}_{\lambda}^{i}\right) \widehat{x}_{\lambda}^{i}}{\lambda_{h} \nabla u_{h}\left(\widehat{x}_{\lambda}^{h}\right) \widehat{x}_{\lambda}^{h}} \text { for all } i, h \in I
$$

where $\left(\widehat{x}_{\lambda}, \widehat{z_{\lambda}}\right)$ is a maximizer of $\widehat{F}_{\lambda}(w)$ over $\widehat{L}(G)$. By Lemma $6,\left(\widehat{x}_{\lambda}, \widehat{z_{\lambda}}\right)$ is a maximizer of $\bar{F}_{\lambda}(w)$ and thus it is Pareto optimal with respect to $\sum_{i \in I} w^{i}-G$. By the second welfare 
theorem, there exists a supporting price $\widehat{p} \in \mathbb{R}_{+}^{L}$ s.t. $(\widehat{x}, \widehat{z}, \widehat{p})$ is a competitive equilibrium with transfers. Namely, $\widehat{z}^{j}=\arg \max \widehat{p} z^{j}$ over $z^{j} \in Z_{j}$ for all $j \in J$ and $\widehat{x}^{i}=\arg \max u_{i}\left(x^{i}\right)$ over $\left\{x^{i} \in \mathbb{R}_{+}^{m} \mid \widehat{p} x^{i} \leq \widehat{p} \widehat{x}^{i}\right\}$ for all $i \in I$. Hence, for some $c$,

$$
c \widehat{p}=\lambda_{i} \nabla u_{i}\left(\widehat{x}^{i}\right) \text { for all } i \in I
$$

Following arguments similar to Proposition 1, prices equal the marginal value of the goods (see once again Mas-Colell et al (1995))

$$
\frac{\partial \bar{F}_{\lambda}}{\partial w_{l}^{i}}(w)=c \widehat{p}_{l}
$$

By (27), (28) and (29),

$$
\frac{\widehat{p} \widehat{x}_{\lambda}^{i}}{\widehat{p} w^{i}}=\alpha
$$

where $\alpha$ is a constant (that does not depend on $i$ ). By (30),

$$
\widehat{p} \sum_{i \in I} \widehat{x}_{\lambda}^{i}=\alpha \widehat{p} \sum_{i \in I} w^{i}=\alpha \widehat{p} W
$$

Since $\sum_{i \in I} \widehat{x}_{\lambda}^{i}=W-G+\sum_{j \in J} \widehat{z}^{j}$,

$$
\alpha=1-\frac{\widehat{p} G}{\widehat{p} W}
$$

By (30) and (31),

Since

$$
\widehat{p} \widehat{x}_{\lambda}^{i}=\widehat{p} w^{i}-\widehat{p} G \frac{\widehat{p} w^{i}}{\widehat{p} W}
$$

$$
\widehat{p} \widehat{x}^{i}=\widehat{p} w^{i}-\widehat{p} \widehat{\tau}^{i}
$$

we have

$$
\widehat{p} \widehat{\tau}^{i}=\frac{\widehat{p} w^{i}}{\widehat{p} W} \widehat{p} G
$$

\section{Appendix B: Optimal Taxation Model}

This Appendix provides a detailed presentation of the model used in section 6.2 and the analytical derivations in the special case discussed in section 6.1.

\section{The Model}

The problem of a household of type $i$ is ${ }^{33}$

$$
\begin{aligned}
& \max _{\left\{c_{i 1}, c_{i 2}, k_{i 1}, n_{i 1}, n_{i 2}\right\}} u\left(c_{i 1}, c_{i 2}, X_{i 1}-n_{i 1}, X_{i 2}-n_{i 2}\right) \text { s.t. } \\
c_{i 1}+k_{i 1}= & R_{1} k_{i 0}+\omega_{1} n_{i 1}-T_{i 1} \\
c_{i 2}= & R_{2} k_{i 1}+\omega_{2} n_{i 2}-T_{i 2} \\
0 \leq & n_{i t} \leq X_{i t} t=1,2 \text { and } c_{i t} \geq 0, t=1,2
\end{aligned}
$$

\footnotetext{
${ }^{33}$ Inada conditions on utility and production allow us to ignore non-negativity constraints. In the main text we also assume utility is independent of $n_{i t}$ so $n_{i t}=X_{i t}$ follows.
} 
where $T_{i t}$ represents the tax bill in each period to be specified below. Firm production functions are given in (12) and lead to capital and labor demand functions which equate the input prices to their marginal product

$$
\begin{aligned}
\omega_{t} & =(1-\theta) K_{t-1}^{\theta} N_{t}^{-\theta} \\
R_{t} & =\theta K_{t-1}^{\theta-1} N_{t}^{1-\theta}
\end{aligned}
$$

for $t=1,2$. In equilibrium $R_{t} K_{t-1}+\omega_{t} N_{t}=Y_{t}$ due to constant returns, which is why specifying household shares $\theta^{i}$ is not needed. The government needs to raise exogenous revenue $G_{t}$ in period $t$

$$
G_{t}=\sum_{i=1}^{2} \pi_{i} T_{i t}
$$

Finally, all markets clear in each period $t=1,2$. Market clearing for capital requires that the firm's demand for capital equals aggregate supply

$$
K_{t}=\pi_{1} k_{1 t}+\left(1-\pi_{1}\right) k_{2 t}
$$

Similarly, the firm's labor demand should equal the aggregate supply

$$
\begin{aligned}
& N_{1}=\pi_{1} n_{11}+\left(1-\pi_{1}\right) n_{21} \\
& N_{2}=\pi_{1} n_{12}+\left(1-\pi_{1}\right) n_{22}
\end{aligned}
$$

By Walras' Law, goods market clearing is also satisfied

$$
C_{t}+K_{t}+G_{t}=Y_{t}
$$

where $C_{t}=\sum_{i=1}^{2} \pi_{i} c_{i t}$ denotes aggregate consumption in period $t$.

We consider two cases distinguished by the government's revenue requirements and available tax instruments. The analytical example discussed in section 6.1 assumes $G_{2}=0$, $T_{i 2}=0, T_{i 1}=\tau_{i}$ i.e. revenue $G_{1}$ is only required in the first period and can be raised using type-specific lump sum taxes. Here tax policy is $\tau=\left(\tau_{1}, \tau_{2}\right)$. The numerical examples in section 6.2 assume instead a revenue need in period two and taxes proportional to capital and labor income, i.e. $G_{1}=0, T_{i 1}=0, T_{i 2}=\tau_{k} R_{2} k_{i 1}+\tau_{n} \omega_{2} n_{i 2}$. In this case, tax policy is $\tau=\left(\tau_{k}, \tau_{n}\right)$.

Let $w$ denote the distribution of initial endowments and $\tau$ denote tax policy. Given $w$ and a feasible $\tau$, the competitive equilibrium consists of household allocations of consumption $c_{i t}^{*}(\tau, w)$, savings $k_{i t}^{*}(\tau, w)$ and labor $n_{i t}^{*}(\tau, w)$ as well as factor prices $R_{t}^{*}(\tau, w), \omega_{t}^{*}(\tau, w)$ for each period $t$. The Ramsey government chooses $\tau$ to maximize social welfare, given by a weighted average of the individual utilities and a set of welfare weights $\left(\lambda_{i}\right)_{i=1}^{2}$, with $\lambda_{i} \geq 0$ and $\lambda_{1} \pi_{1}+\lambda_{2} \pi_{2}=1$. Thus, for a given set of weights $\left(\lambda_{i}\right)_{i=1}^{2}$, the problem is to choose feasible tax policy $\tau$ so as to maximize

$$
F_{\lambda}(w)=\max _{\tau} \sum_{i=1}^{2} \lambda_{i} \pi_{i} u\left(c_{i 1}^{*}(\tau, w), X_{i 1}-n_{i 1}^{*}(\tau, w), c_{i 2}^{*}(\tau, w), X_{i 2}-n_{i 2}^{*}(\tau, w)\right)
$$


The maximizer of this social optimization problem $\tau^{R A}(\lambda, w)$ depends on the weights $\lambda$ and the distribution of endowments $w$. It also defines Ramsey allocations denoted similarly, $c_{i 1}^{R A}(w, \lambda), c_{i 2}^{R A}(w, \lambda)$ and so on. Partial differentiation of the maximized value (SWF) with respect to each element of $w$ gives the gradient of the SWF evaluated at $w$. The inner product of this gradient with each individual's $\tilde{w}_{i}$ gives the social contribution of the individual. In a similar fashion, the private contribution of the Ramsey allocation to an individual's utility $u(\cdot)$ can be computed. Finally, the VP principle in equation (2) provides an equation which, together with $\lambda_{1} \pi_{1}+\lambda_{2} \pi_{2}=1$, can be solved for the VP weights $\lambda^{V P}$. VP allocations and taxes follow by direct substitution of $\lambda^{V P}$ in $\tau^{R A}(\lambda, w), c_{i 1}^{R A}(w, \lambda), c_{i 2}^{R A}(w, \lambda)$ etc.

In general, all of these computations, i.e. solving for the competitive equilibrium allocations and prices, solving for Ramsey allocations and taxes, evaluating the SWF and its derivatives and solving for the VP weights, are done numerically. This is what we do for the results of Section 6.2. Below we illustrate the idea by going through these computations analytically in the special case of logarithmic utility and lump sum taxation.

\section{Logarithmic utility and lump sum taxation.}

In this case, tax policy consists of a pair $\left(\tau_{1}, \tau_{2}\right)$. Equation (37) defines feasible tax policies and essentially implies that only one of the two taxes is a free choice for the government, while the second is fully determined by the feasibility condition. Thus, we will refer to $\tau_{1}$ only as the tax policy in what follows. Let the individual and aggregate incomes in period one be denoted as $i n c_{i 1}=R_{1} k_{i 0}+\omega_{1} n_{i 1}$ and $I N C_{1}=R_{1} K_{0}+\omega_{1} N_{1}$. Let $w^{i}=\left(0,0, \pi_{i} k_{i 0}, 0, \pi_{i} n_{i 1}, \pi_{i} n_{i 2}\right)$ be the initial endowments of the representative of type $i .^{34}$ Given $w$ and a tax policy $\tau_{1}$, the competitive equilibrium can be computed explicitly because with $\log$ utility the aggregate capital $K_{1}$ and the factor prices $R_{t}, \omega_{t}$ are independent of tax policy and given by

$$
\begin{aligned}
K_{1} & =\frac{\beta \theta}{1+\beta \theta}\left[I N C_{1}-G_{1}\right] \\
R_{t} & =\theta K_{t-1}^{\theta-1} N_{t}^{1-\theta}, t=1,2 \\
\omega_{t} & =(1-\theta) K_{t-1}^{\theta} N_{t}^{-\theta}, t=1,2
\end{aligned}
$$

Individual consumption and savings for $i=1,2$ are given by

$$
\begin{aligned}
k_{i 1}^{*}\left(\tau_{1}, w\right) & =\frac{\beta}{1+\beta}\left(i n c_{i 1}-\tau_{i}\right)-\frac{\beta(1-\theta)}{(1+\beta)(1+\beta \theta)} \frac{n_{i 2}}{N_{2}}\left[I N C_{1}-G_{1}\right] \\
c_{i 1}^{*}\left(\tau_{1}, w\right) & \left.=\frac{1}{1+\beta}\left(i n c_{i 1}-\tau_{i}\right)+\frac{\beta(1-\theta)}{(1+\beta)(1+\beta \theta)} \frac{n_{i 2}}{N_{2}}\left[I N C_{1}-G_{1}\right]\right] \\
c_{i 2}^{*}\left(\tau_{1}, w\right) & =A K_{1}^{\theta} N_{2}^{1-\theta}\left[\frac{1+\beta \theta}{1+\beta}\left(\frac{i n c_{i 1}-\tau_{i}}{I N C_{1}-G_{1}}\right)+\frac{\beta(1-\theta)}{1+\beta} \frac{n_{i 2}}{N_{2}}\right]
\end{aligned}
$$

where

$$
\tau_{2}=\frac{1}{1-\pi_{1}} G_{1}-\frac{\pi_{1}}{1-\pi_{1}} \tau_{1}
$$

In this example, the only effect of tax policy is to redistribute initial wealth so that the initial distribution of income $\left(i n c_{11}, i n c_{21}\right)$ can be changed through taxes to the new

\footnotetext{
${ }^{34}$ Strictly speaking, the endowment of time is $X_{i t}$ not $n_{i t}$, but with inelastic labor supply the two are the same so we use $n_{i t}$ throughout the example.
} 
distribution $\left(i n c_{11}-\tau_{1}, i n c_{21}-\tau_{2}\right)$. In particular, the government can potentially use taxes to wipe out any initial wealth heterogeneity, i.e. engage in perfect redistribution, without affecting aggregates. In the Ramsey problem defined in (41) the amount of redistribution that will optimally take place will depend on the welfare weights, which determine the strength of the redistribution motive. Using the logarithmic utility in (16) the first order condition with respect to $\tau_{1}$ is

$$
\sum_{i=1}^{2} \lambda_{i} \pi_{i}\left[\frac{1}{c_{i 1}} \frac{\partial c_{i 1}^{*}\left(\tau_{1}, w\right)}{\partial \tau_{1}}+\beta \frac{1}{c_{i 2}} \frac{\partial c_{i 2}^{*}\left(\tau_{1}, w\right)}{\partial \tau_{1}}\right]=0
$$

and using equilibrium relations this can be simplified to

$$
\lambda_{1} \frac{1}{c_{i 1}^{*}\left(\tau_{1}, w\right)}=\lambda_{2} \frac{1}{c_{i 2}^{*}\left(\tau_{1}, w\right)}
$$

Using (43) and (44) the optimal Ramsey tax can be solved for as a function of weights $\lambda$ and endowments $w$.

$$
\tau_{1}(\lambda, w)=i n c_{11}-\frac{1+\beta}{1+\beta \theta}\left[\lambda_{1}-\frac{\beta(1-\theta)}{1+\beta} \frac{n_{12}}{N_{2}}\right]\left[I N C_{1}-G_{1}\right]
$$

Using the government budget constraint the other $\operatorname{tax} \tau_{2}(\lambda, w)$ is given by:

$$
\tau_{2}(\lambda, w)=\frac{G_{1}-\pi_{1} \tau_{1}(\lambda, w)}{1-\pi_{1}}=i n c_{12}-\frac{1+\beta}{1+\beta \theta}\left[\lambda_{2}-\frac{\beta(1-\theta)}{1+\beta} \frac{n_{22}}{N_{2}}\right]\left[I N C_{1}-G_{1}\right]
$$

Finally, using (42)-(46) we can derive the allocations as functions of $(\lambda, w)$

$$
\begin{aligned}
k_{i 1}^{R A}(\lambda, w) & =\lambda_{i} \frac{\beta}{1+\beta \theta}\left[I N C_{1}-G_{1}\right]-\frac{\beta(1-\theta)}{(1+\beta \theta)} \frac{n_{i 2}}{N_{2}}\left[I N C_{1}-G_{1}\right] \\
c_{i 1}^{R A}(\lambda, w) & =\frac{\lambda_{i}}{1+\beta \theta}\left[I N C_{1}-G_{1}\right] \\
c_{i 2}^{R A}(\lambda, w) & =\lambda_{i} A K_{1}^{\theta} N_{2}^{1-\theta}
\end{aligned}
$$

The previous equations clearly illustrate that if $\lambda_{1}=\lambda_{2}=1$, i.e. if we use a social welfare function that assigns equal weights, then all of the initial wealth heterogeneity will be wiped out by the optimal tax and agents will become identical. To put it differently, equal weights prescribes perfect redistribution in the absence of other motives for taxation. Using $n_{i t}=N_{t}=X$ for all $i, t$, the tax in this case is

$$
\begin{aligned}
\tau_{1}(1, w) & =G_{1}+i n c_{i 1}-I N C_{1} \\
& =G_{1}+R_{1} K_{0}\left(\frac{k_{10}}{K_{0}}-1\right)
\end{aligned}
$$

The VP weights can be computed endogenously by evaluating the social contributions of individuals for any $\lambda$. After simplifying, the social welfare function can be written as:

$$
F_{\lambda}(w) \equiv \sum_{i=1}^{2} \lambda_{i} \pi_{i}\left[\log \left(\frac{\lambda_{i}}{1+\beta \theta}\right)+\beta \log \left(\lambda_{i}\right)\right]+\log \left(I N C_{1}-G_{1}\right)+\beta \log \left(A K_{1}^{\theta} N_{2}^{1-\theta}\right)
$$


To determine the contribution of type $i$ through his initial endowments we need to compute first the partial derivatives of the welfare function, which is a function of the distribution of endowments $w$. Since the only positive endowments of an individual are $k_{i 0}, X_{i 1}\left(=n_{i 1}\right)$ and $X_{i 2}\left(=n_{i 1}\right)$ we only need to compute three derivatives ${ }^{35}$

$$
\begin{aligned}
\frac{\partial F_{\lambda}(w)}{\partial\left(\pi_{i} k_{i 0}\right)} & =\frac{1+\beta \theta}{I N C_{1}-G_{1}} R_{1} \\
\frac{\partial F_{\lambda}(w)}{\partial\left(\pi_{i} k_{i 0}\right)} & =\frac{1+\beta \theta}{I N C_{1}-G_{1}} \omega_{1} \\
\frac{\partial F_{\lambda}(w)}{\partial\left(\pi_{i} k_{i 0}\right)} & =\frac{1+\beta \theta}{I N C_{1}-G_{1}} \frac{\omega_{2}}{R_{2}}
\end{aligned}
$$

The contribution of these endowments to the SWF at $w$ is then

$$
C\left(F_{\lambda}, \tilde{w}^{i}, w\right)=\frac{1+\beta \theta}{I N C_{1}-G_{1}} \pi_{i}\left[R_{1} k_{i 0}+\omega_{1} n_{i 1}+\frac{\omega_{2}}{R_{2}} n_{i 2}\right]
$$

Next, we compute the contribution of the individual bundle of goods to the individual welfare function $\lambda_{i} \pi_{i} U_{i}\left(c^{i}\right)=\lambda_{i} \pi_{i}\left[\log \left(c_{i 1}\right)+\beta \log \left(c_{i 2}\right)\right]$. Note that the individual welfare function only depends on the two consumption goods and evaluating derivatives is trivial. The contribution of the final allocation to $i$ 's welfare function is

$$
C\left(\lambda_{i} \pi_{i} U, x_{\lambda}^{i}\right)=c_{i 1}^{R A}(w, \lambda) \lambda_{i} \pi_{i} \frac{1}{c_{i 1}^{R A}(w, \lambda)}+c_{i 2}^{R A}(w, \lambda) \lambda_{i} \pi_{i} \beta \frac{1}{c_{i 2}^{R A}(w, \lambda)}
$$

The condition for VP weights is given by:

$$
\frac{C\left(\lambda_{1} \pi_{1} U, x_{\lambda}^{1}\right)}{C\left(\lambda_{2} \pi_{2} U, x_{\lambda}^{2}\right)}=\frac{C\left(F_{\lambda}, \tilde{w}^{1}, w\right)}{C\left(F_{\lambda}, \tilde{w}^{2}, w\right)}
$$

By (47) and (48)

$$
\frac{c_{11} \lambda_{1} \frac{1}{c_{11}}+c_{12} \lambda_{1} \beta \frac{1}{c_{12}}}{c_{21} \lambda_{2} \frac{1}{c_{21}}+c_{22} \lambda_{2} \beta \frac{1}{c_{22}}}=\frac{R_{1} k_{10}+\omega_{1} n_{11}+\frac{\omega_{2}}{R_{2}} n_{12}}{R_{1} k_{20}+\omega_{1} n_{21}+\frac{\omega_{2}}{R_{2}} n_{22}}
$$

The VP weights in equation (17) follow immediately. If, instead we use the household's Euler equation $\beta \frac{1}{c_{i 2}}=\frac{1}{R_{2}} \frac{1}{c_{i 1}}$ and the Ramsey condition $\lambda_{1} \frac{1}{c_{11}}=\lambda_{2} \frac{1}{c_{21}}$ we have

$$
\frac{c_{11}+\frac{1}{R_{2}} c_{12}}{c_{21}+\frac{1}{R_{2}} c_{22}}=\frac{R_{1} k_{10}+\omega_{1} n_{11}+\frac{\omega_{2}}{R_{2}} n_{12}}{R_{1} k_{20}+\omega_{1} n_{21}+\frac{\omega_{2}}{R_{2}} n_{22}}
$$

The sequential budget constraints of type $i$ imply the following (date- 0 ) budget:

$$
c_{i 1}+\frac{1}{R_{2}} c_{i 2}+\tau_{i}=R_{1} k_{i 0}+\omega_{1} n_{i 1}+\frac{\omega_{2}}{R_{2}} n_{i 2}
$$

Together with (49), this implies that the VP tax is given by equation (15).

\footnotetext{
${ }^{35}$ We compute the marginal change in $\pi_{i} k_{i 0}$ to capture a change in one individual's endowment as opposed to all individuals of type $i$.
} 


\section{REFERENCES}

[1] Abraham, A., Carceles-Poveda, E., 2010. Endogenous Trading Constraints with Incomplete Asset Markets. Journal of Economic Theory, 145, pp. 974-1004.

[2] Aiyagari, S.R., 1994. Uninsurable Idiosyncratic Risk and Aggregate Saving. Quarterly Journal of Economics, 109(3), pp. 659-684.

[3] Aiyagari, S.R., 1995. Optimal Capital Income Taxation with Incomplete Markets, Borrowing Constraints and Constant Discounting. Journal of Political Economy 103(6), pp. 1158-75.

[4] Anagnostopoulos, A., Carceles-Poveda, E., Lin, D., 2012. Dividend and Capital Gains Taxation under Incomplete Markets. Journal of Monetary Economics, vol. 59 (7), pp. 599-611.

[5] Aumann, R., 1975. Values of markets with a continuum of traders, Econometrica vol. 43 (4), pp. 611-646.

[6] Aumann, R., Shapley, L.S., 1974. Values of Non-Atomic Games, Princeton University Press, Princeton, New Jersey.

[7] Benabou, R., 2002. Tax and Education Policy in a Heterogeneous-Agent Economy: What Levels of Redistribution Maximize Growth and Efficiency?, Econometrica, 7 (2), pp. 481-517.

[8] Davila, J., Hong, J.H., Krusell, P., Ríos-Rull, J.V., 2012. Constrained Efficiency in the Neoclassical Growth Model with Uninsurable Idiosyncratic Shocks. Econometrica vol. 80 (6), pp. 2431-2467.

[9] Domeij, D., Heathcote, J., 2004. On the Distributional Effects of Reducing Capital Taxes. International Economic Review 45(2), pp. 523-554.

[10] Fleurbaey M., Maniquet, F., 2014. Optimal Taxation Theory and Principles of Fairness. Core Discussion Paper.

[11] Harsanyi, J. C, 1953. Cardinal Utility in Welfare Economics and in the Theory of RiskTaking, Journal of Political Economy, vol. 61 (5), pp. 434-435.

[12] Harsanyi, J. C, 1955. Cardinal Welfare, Individualistic Ethics, and the Interpersonal Comparison of Utility, Journal of Political Economy, vol. 63 (4), pp. 309-321.

[13] Heathcote, J., Storesletten, K, Violante, G. L., 2014. Optimal Tax Progressivity: An Analytical Framework, working paper.

[14] Kehoe P., and Perri F., 2002. International Business Cycles With Endogenous Incomplete Markets, Econometrica, 70, vol. 3, pp. 907-928.

[15] Mankiw, G. N., 2010. Spreading the Wealth Around: Reflection Inspired by Joe the Plumber, Eastern Economic Journal, 36, pp. 285-298. 
[16] Mas -Colell, A, Whinston, M. D., Green, J. R., 1995. Microeconomic Theory. Oxford University Press, New York, New York.

[17] Mirman,L., Tauman, Y., 1982. Demand compatible equitable cost sharing prices, Mathematics of Operations Research, vol. 7 (1), pp. 40-56.

[18] Negishi, T., 1960. Welfare Economics and Existence of an Equilibrium for a Competitive Economy, Metroeconomica, vol. 12 (2-3), pp. 92-97.

[19] Noszick, R., 1974. Anarchy, State and Utopia, Basic Books, New York.

[20] Rawls, J., 1971. A Theory of Justice, Belknap Press of Harvard University Press, Cambridge, MA.

[21] Saez, E., Stantcheva, S., 2015. Generalized Social Marginal Welfare Weights for Optimal Tax Theory, working paper.

[22] Samet, D., Tauman, Y., 1982. The determination of marginal cost prices under a set of axioms, Econometrica, vol. 50 (4), pp. 895-909.

[23] Shapley, L.S., 1953. "A Value for n-person Games". In Contributions to the Theory of Games, volume II, ed. by H.W. Kuhn and A.W. Tucker, Princeton, NJ: Princeton University Press, pp. 307-317.

[24] Shapley, L.S., 1969. Utility Comparison and the Theory of Games. In "La Decision Aggregation Ordres de Preference", Paris, Editions du Centre National de la Recherche Scientifique, 251-263.

[25] Weinstein, J., 2011. Fairness and Tax Policy: A Response to Mankiw's Proposed "Just Deserts", Eastern Economic Journal, 37, 313-320.

[26] Weinzierl, M., 2014. The Promise of Positive Optimal Taxation: Normative Diversity and a Role for Equal Sacrifice, Working Paper, Harvard Business School.

[27] Young, H. P., 1988. Distributive Justice in Taxation, Journal of Economic Theory, vol. 44 (2), pp. 321-335.

[28] Young, H. P., 1990. Progressive Taxation and Equal Sacrifice, American Economic Review, vol 80 (1), pp. 253-266. 